\title{
NEOWISE REACTIVATION MISSION YEAR ONE: PRELIMINARY ASTEROID DIAMETERS AND ALBEDOS
}

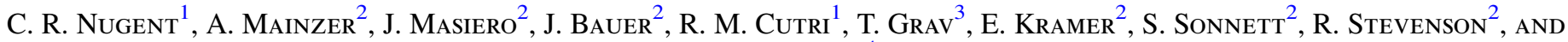 \\ E. L. WRIGHT \\ ${ }^{1}$ Infrared Processing and Analysis Center, California Institute of Technology, Pasadena, CA 91125, USA; cnugent@ipac.caltech.edu \\ 2 Jet Propulsion Laboratory, California Institute of Technology, Pasadena, CA 91109, USA \\ ${ }^{3}$ Planetary Science Institute, Tucson, AZ, USA \\ ${ }^{4}$ Department of Physics and Astronomy, University of California, Los Angeles, CA 90095, USA \\ Received 2015 June 11; accepted 2015 September 4; published 2015 November 24
}

\begin{abstract}
We present preliminary diameters and albedos for 7956 asteroids detected in the first year of the NEOWISE Reactivation mission. Of those, 201 are near-Earth asteroids and 7755 are Main Belt or Mars-crossing asteroids. $17 \%$ of these objects have not been previously characterized using the Near-Earth Object Wide-field Infrared Survey Explorer, or "NEOWISE" thermal measurements. Diameters are determined to an accuracy of 20\% or better. If good-quality $H$ magnitudes are available, albedos can be determined to within $\sim 40 \%$ or better.
\end{abstract}

Key words: minor planets, asteroids: general

Supporting material: machine-readable tables

\section{INTRODUCTION}

Sizes and albedos of asteroids are basic quantities that can be used to answer a range of scientific questions. A significant number of diameter measurements produce a size-frequency distribution, which can constrain models of asteroid formation and evolution (Zellner 1979; Gradie \& Tedesco 1982; Bus \& Binzel 2002; Tedesco et al. 2002; Masiero et al. 2011). Asteroid albedos aid the identification of collisional family members (Carruba et al. 2013; Masiero et al. 2013, 2015; Walsh et al. 2013; Milani et al. 2014), and allow for basic characterization of asteroid composition (Mainzer et al. 2011c; Grav et al. 2012a; Masiero et al. 2014).

Most observations of asteroids are made in visible wavelengths, where flux is dependent on both size and albedo. Observations in other wavelengths, such as the infrared (e.g., Hansen 1976; Cruikshank 1977; Lebofsky et al. 1978; Morrison \& Lebofsky 1979; Delbó et al. 2003, 2011; Wolters et al. 2005, 2008; Matter et al. 2011; Müller et al. 2012, 2013) or radio (e.g., Ostro et al. 2002; Benner et al. 2015), are needed to determine these quantities precisely. At present, well-determined diameters and albedos have been measured for less than a quarter of known asteroids.

The infrared NEOWISE project (Mainzer et al. 2011a) has measured diameters and albedos for $\sim 20 \%$ of the known asteroid population, the majority of these measurements to date (Grav et al. 2011, 2012c; Mainzer et al. 2011b, 2012, 2015; Masiero et al. 2011, 2012; Bauer et al. 2013). Here, we expand the number of asteroids characterized by NEOWISE, deriving diameters and albedos for asteroids detected by NEOWISE between 2013 December 13 and 2014 December 13 during the first year of the Reactivation mission.

The NEOWISE mission uses the Wide-field Infrared Survey Explorer (WISE) spacecraft, which images the entire sky using freeze-frame scanning from a Sun-synchronous polar orbit (Wright et al. 2010; Cutri et al. 2012). WISE is equipped with a $50 \mathrm{~cm}$ telescope and four $1024 \times 1024$ pixel focal plane array detectors that simultaneously image the same $47 \times 47$ arcmin field of view in $3.4,4.6,12$, and $22 \mu \mathrm{m}$ bands, all originally cooled by solid hydrogen cryogen. WISE scans the sky between the ecliptic poles continuously during its 95 minute orbit. A tertiary scan mirror freezes the sky on the focal planes for $11 \mathrm{~s}$ while the detectors are read out, producing a sequence of adjacent images with $7.7 \mathrm{~s}$ exposure times in the 3.4 and $4.6 \mu \mathrm{m}$ bands and $8.8 \mathrm{~s}$ in the 12 and $22 \mu \mathrm{m}$ bands. The orbit precesses at an average rate of approximately one degree per day, so that the full sky is covered in six months.

WISE was launched on 2009 December 14 and began surveying on 2010 January 7. WISE scanned the sky 1.5 times during the 9.5 months while it was cooled by its hydrogen cryogen. After the hydrogen was depleted, the survey continued as NEOWISE until 2011 February 1, using the 3.4 and $4.6 \mu \mathrm{m}$ detectors that operated at near full sensitivity with purely passive cooling. During the additional four months of "post-cryo" operations, coverage of the entire inner Main Asteroid Belt was completed, along with a second complete coverage of the sky. WISE/NEOWISE was placed into hibernation in 2011 mid-February. In this mode, the solar panels were held facing the Sun and the telescope pointed toward the north ecliptic pole. The telescope viewed the Earth during half of each orbit, resulting in some heating.

The WISE spacecraft was brought out of hibernation in 2013 September and renamed NEOWISE to continue its mission to discover, track, and characterize asteroids through $\sim 2017$ (Mainzer et al. 2014). The telescope was restored to near zenith pointing, which enabled the optics and focal planes to cool passively back to $\sim 74$ K. Survey operations resumed on 2013 December 13, with the 3.4 and $4.6 \mu \mathrm{m}$ detectors operating at a sensitivity comparable to that during the original WISE cryogen survey (Cutri et al. 2015). The NEOWISE moniker, an acronym of near-Earth object WISE, encompasses both the archiving of individual images to allow for the detection of transient objects, and the extensions of the mission beyond WISE's original 9-month lifetime.

NEOWISE uses the same survey and observing strategy as the original WISE mission (Wright et al. 2010). The majority of each orbit is devoted to observations, with only brief breaks for data transmission and momentum unloading. The spacecraft carries a body-fixed antenna, and therefore must reorient itself to communicate with the Tracking and Data Relay Satellite System, which relays the data to Earth. Data transmission is 


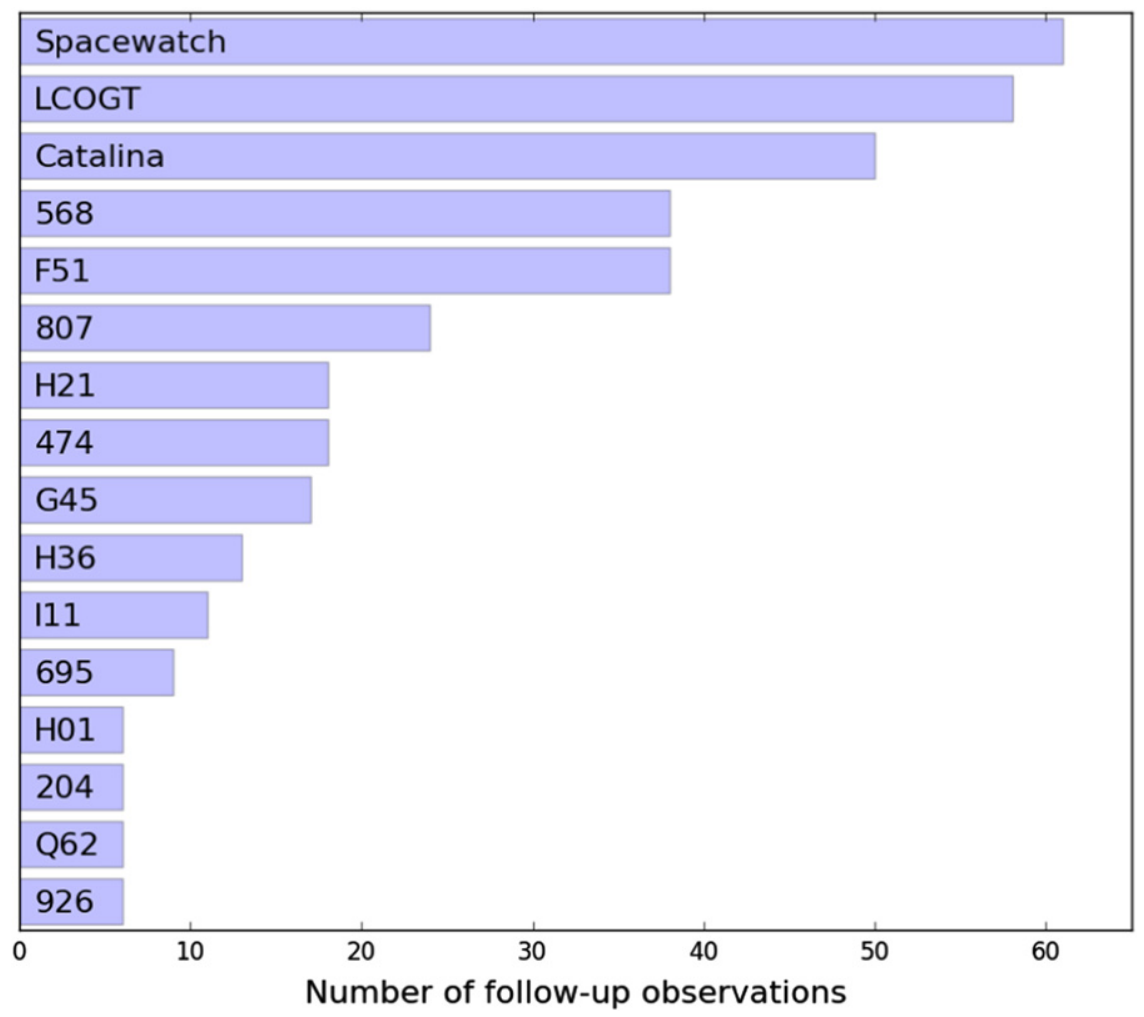

Figure 1. Number of follow-up observations by observatories that contributed $>5$ observations during the Year 1 Reactivation. Spacewatch, LCOGT, and Catalina employ multiple telescopes; their observatory codes have been grouped together. Observatory code 568, Mauna Kea, is frequently used by the Tholen group.

timed to only interrupt survey coverage near the ecliptic poles, which are observed frequently. Momentum unloading, which can result in streaked images, is also completed at this time.

Data processing for NEOWISE uses the WISE Science Data System (Cutri et al. 2015), which performs instrumental, photometric, and astrometric calibration for each individual set of 3.4 and $4.6 \mu \mathrm{m}$ exposures obtained by the spacecraft, and detects and characterizes sources on each exposure. The calibrated images and the database of positions and fluxes of sources extracted from those images for the first year of NEOWISE survey observations were released in 2015 March (Cutri et al. 2015).

The WISE Moving Object Pipeline System (WMOPS; Cutri et al. 2012) identifies sources that display motion between the different observations of the same region on the sky. WMOPS uses the extracted source lists from sets of images to first identify and filter out sources that appear stationary between individual exposures, and then links non-stationary detections into sets that exhibit physically plausible motion on the sky. Generally, objects within 70 AU of the Sun move quickly enough to be detected by WMOPS (Mainzer et al. 2011a, see also Bauer et al. 2013). Those candidate moving objects that are not associated with known asteroids, comets, planets, or planetary satellites are verified individually by NEOWISE scientists. A minimum of five independent detections are required for a tracklet (a set of position/time pairs) to be considered reliable. Tracklets for each verified new candidate object and previously known solar system objects are reported to the IAU Minor Planet Center (MPC) three times per week. The MPC performs initial orbit determination, associates the NEOWISE tracklets with known objects, and archives the NEOWISE astrometry and times in its observation database.
Candidates confirmed by the MPC to be possible new nearEarth-objects (NEOs) are posted to their NEO Confirmation page for prompt follow-up observations by ground-based observers. Rapid follow-up is essential for NEOWISE NEO candidates because the NEOWISE arcs are usually short, and the asteroid's projected positional uncertainties grow quickly, making reliable recovery difficult after 2-3 weeks. To ensure prompt follow-up, NEOWISE observations are reported to the MPC less than three days after observations on board the spacecraft. A NEOWISE candidate discovery has a minimum of five observations over $\sim 3 \mathrm{hr}$, although typical objects have $\sim 12$ observations spanning $\sim 1.5$ days.

Targets observed by NEOWISE can pose unique challenges to ground-based follow-up observers. NEOWISE's orbit allows observations to be made at all declinations, and observing is independent of lunar phase. Ground-based observers are limited to a fixed declination range, and must sometimes deal with light from the moon and terrestrial weather, which can preclude observations. Moreover, NEOWISE discoveries are frequently extremely dark (see Figure 5), often requiring 2-4 m class telescopes to detect them at low solar elongations.

Observers around the globe (including both amateurs and professionals) have contributed essential follow-up observations, which are defined here as an observation of an object within 15 days of its first observation on board the spacecraft. Significant contributors of follow-up observations are given in Figure 1. The Spacewatch Project (McMillan 2007) contributes a large share of recoveries in the northern hemisphere. The Las Cumbres Observatory Global Telescope (LCOGT) Network of robotically operated queue-scheduled telescopes (Brown et al. 2013) has been an extremely useful resource for securing detections when weather is poor at a particular site. 
Additionally, the group led by D. Tholen using the University of Hawaii $2.2 \mathrm{~m}$ and Canada-France-Hawaii $4 \mathrm{~m}$ telescopes has successfully detected the targets with the faintest optical magnitudes in the northern hemisphere (e.g., Tholen et al. 2014). The NEOWISE team was awarded time with the DECam instrument on the Cerro Tololo Inter-American Observatory $4 \mathrm{~m}$ telescope, which has proven invaluable for the recovery of low albedo objects at extreme declinations in the southern hemisphere.

We present diameters and albedos for 201 near-Earth asteroids (NEAs) and 7755 Main Belt and Mars-crossing asteroids detected in the first year of reactivation, between 2013 December 13 and 2014 December 13. This includes the 38 NEAs discovered by NEOWISE during those dates.

\section{METHODS}

\subsection{Observations}

The MPC is responsible for verifying and archiving asteroid astrometry. To obtain the verified record of objects found by the WMOPS pipeline in the NEOWISE data, we queried the MPC observations files "NumObs.txt" and "UnnObs.txt" for NEOWISE (observatory code C51) observations between 2013 December 13 and 2014 December 13. This returned the list of object identifications, along with the observation times and NEOWISE measured astrometry. This included known objects and WMOPS asteroid discoveries made during that time.

The NASA/IPAC Infrared Science Archive (IRSA, at http://irsa.ipac.caltech.edu) NEOWISE-R Single Exposure Source Table was then queried for the fluxes of sources detected in the NEOWISE data. The list of detections extracted from the MPC files was converted into GATOR format (see http://irsa.ipac.caltech.edu/applications/Gator/

GatorAid/irsa/QuickGuidetoGator.htm), and uploaded into the IRSA interface using a cone search radius of 2 arcsec and a restriction that times match the MPC-archived observation time to within $2 \mathrm{~s}$. This two-step process of querying both the MPC archive and the NEOWISE-R Single Exposure (L1b) Source Table ensures that only detections verified both by the NEOWISE object identification routines and the MPC are used for thermal modeling. While there may be additional objects in the database that were detected fewer than five times, or are just below the single-frame detection threshold, this method of extracting moving object detections ensures high reliability, since WMOPS actively works to exclude fixed sources such as stars and galaxies from tracklets. Sources with fewer than five detections or those that fall just below the single-frame detection threshold will be extracted in future processing.

NEOWISE detections were further filtered using several measurement and image quality flags. We required detections to have "ph_qual" values of "A," "B," or "C," "cc_flag" values of " 0 ," and "qual_frame" values of " 10 ." The "ph_qual" flag represents photometric quality, accepting a value of " $\mathrm{C}$ " or higher ensures that the sources was detected with a flux signalto-noise ratio $>2$. The "cc_flag," or contamination and confusion flag, indicates whether the source measurement may be compromised due to a nearby image artifact. By filtering for "cc_flag" $=0$, we select for sources unaffected by known artifacts. Finally, "qual_frame" is an overall quality grade for the entire image in which the source was detected. We accepted only the best-quality images, those with a score of " 10. ."
Table 1

NEOWISE Magnitudes for the NEAs Modeled in This Paper

\begin{tabular}{|c|c|c|c|c|}
\hline Name & MJD & $W 1$ (mag) & $W 2$ (mag) & Aperture \\
\hline 01566 & 56795.5373147 & $>16.339$ & $13.317 \pm 0.086$ & 0 \\
\hline 01566 & 56795.668982 & $15.340 \pm 0.132$ & $13.287 \pm 0.104$ & 0 \\
\hline 01566 & 56795.8005219 & $15.270 \pm 0.133$ & $13.255 \pm 0.157$ & 0 \\
\hline 01566 & 56795.8663555 & $15.268 \pm 0.137$ & $13.226 \pm 0.125$ & 0 \\
\hline 01566 & 56795.9321892 & $15.590 \pm 0.200$ & $13.395 \pm 0.166$ & 0 \\
\hline 01566 & 56796.1295626 & $14.904 \pm 0.097$ & $13.348 \pm 0.102$ & 0 \\
\hline 01566 & 56796.2612299 & $15.829 \pm 0.192$ & $13.467 \pm 0.196$ & 0 \\
\hline 01580 & 56955.905682 & $>16.484$ & $14.033 \pm 0.171$ & 0 \\
\hline 01580 & 56956.037222 & $>16.124$ & $14.230 \pm 0.156$ & 0 \\
\hline 01580 & 56956.431715 & $17.100 \pm 0.538$ & $13.972 \pm 0.136$ & 0 \\
\hline 01580 & 56956.5631277 & $16.951 \pm 0.474$ & $14.158 \pm 0.198$ & 0 \\
\hline 01580 & 56956.6289614 & $>16.168$ & $14.159 \pm 0.157$ & 0 \\
\hline 01580 & 56956.6946677 & $16.178 \pm 0.252$ & $14.312 \pm 0.187$ & 0 \\
\hline 01580 & 56956.7603741 & $16.442 \pm 0.316$ & $13.976 \pm 0.154$ & 0 \\
\hline 01580 & 56956.8262078 & $>17.166$ & $13.988 \pm 0.209$ & 0 \\
\hline 01580 & 56956.8919142 & $16.944 \pm 0.523$ & $14.050 \pm 0.134$ & 0 \\
\hline 01580 & 56956.9576205 & $16.206 \pm 0.291$ & $14.282 \pm 0.186$ & 0 \\
\hline 01580 & 56957.0891606 & $16.795 \pm 0.397$ & $14.271 \pm 0.180$ & 0 \\
\hline 01580 & 56957.4179471 & $>17.009$ & $13.987 \pm 0.145$ & 0 \\
\hline 01620 & 56993.9087248 & $15.427 \pm 0.137$ & $14.075 \pm 0.156$ & 0 \\
\hline 01620 & 56994.3030911 & $15.463 \pm 0.154$ & $14.049 \pm 0.200$ & 0 \\
\hline 01620 & 56994.434504 & $15.420 \pm 0.144$ & $13.556 \pm 0.102$ & 0 \\
\hline 01620 & 56994.5659171 & $15.596 \pm 0.171$ & $14.375 \pm 0.205$ & 0 \\
\hline 01620 & 56994.5660444 & $16.012 \pm 0.212$ & $14.305 \pm 0.221$ & 0 \\
\hline 01620 & 56994.6317509 & $15.754 \pm 0.198$ & $13.846 \pm 0.169$ & 0 \\
\hline 01620 & 56994.7631639 & $15.513 \pm 0.145$ & $13.807 \pm 0.132$ & 0 \\
\hline 01620 & 56994.8945768 & $15.794 \pm 0.216$ & $14.203 \pm 0.228$ & 0 \\
\hline 01620 & 56994.8947042 & $15.843 \pm 0.488$ & $14.106 \pm 0.155$ & 0 \\
\hline 01620 & 56994.9604107 & $15.241 \pm 0.129$ & $13.988 \pm 0.155$ & 0 \\
\hline 01620 & 56995.0918237 & $15.203 \pm 0.109$ & $13.637 \pm 0.140$ & 0 \\
\hline 01620 & 56995.2890705 & $15.354 \pm 0.124$ & $13.483 \pm 0.115$ & 0 \\
\hline 01620 & 56995.4204835 & $15.411 \pm 0.161$ & $13.768 \pm 0.175$ & 0 \\
\hline 01620 & 56995.8147225 & $15.912 \pm 0.223$ & $14.289 \pm 0.205$ & 0 \\
\hline
\end{tabular}

Note. Given are the time of the observation in modified Julian date (MJD), and the magnitude in the $3.4 \mu \mathrm{m}(W 1)$ and $4.6 \mu \mathrm{m}$ bands (W2). Non-detections at a particular wavelength represent $95 \%$ confidence limits (Cutri et al. 2012). The aperture radius in arcseconds used for aperture photometry is given under "Aperture"; " 0 " indicates that the pipeline profile fit photometry was used. Only observations for the first three objects are shown.

(This table is available in its entirety in machine-readable form.)

The filtered data from the NEOWISE Single Exposure Source Table are high-quality source measurements that were found at the times and locations of NEOWISE WMOPS detections submitted to the MPC. To further guard against the possibility of confusing a minor planet with fixed background sources such as stars and galaxies, we uploaded the filtered data to the IRSA catalog query engine, referencing the WISE Allsky Source Catalog to determine if any single-frame detections fell within 6.5 arcsec of an Atlas source. The WISE Source Catalog is generated using multiple independent single exposure images. Fast-moving solar system objects are suppressed during the construction of the catalog. A search radius of 6.5 arcsec was chosen, as it is the approximate size of the WISE beam in the 3.4 and $4.6 \mu \mathrm{m}$ bands.

We required at least three observations with magnitude errors $\sigma_{\text {mag }} \leqslant 0.25$ in one band. The largest main-belt asteroids (MBAs) can saturate the NEOWISE detectors, resulting in 

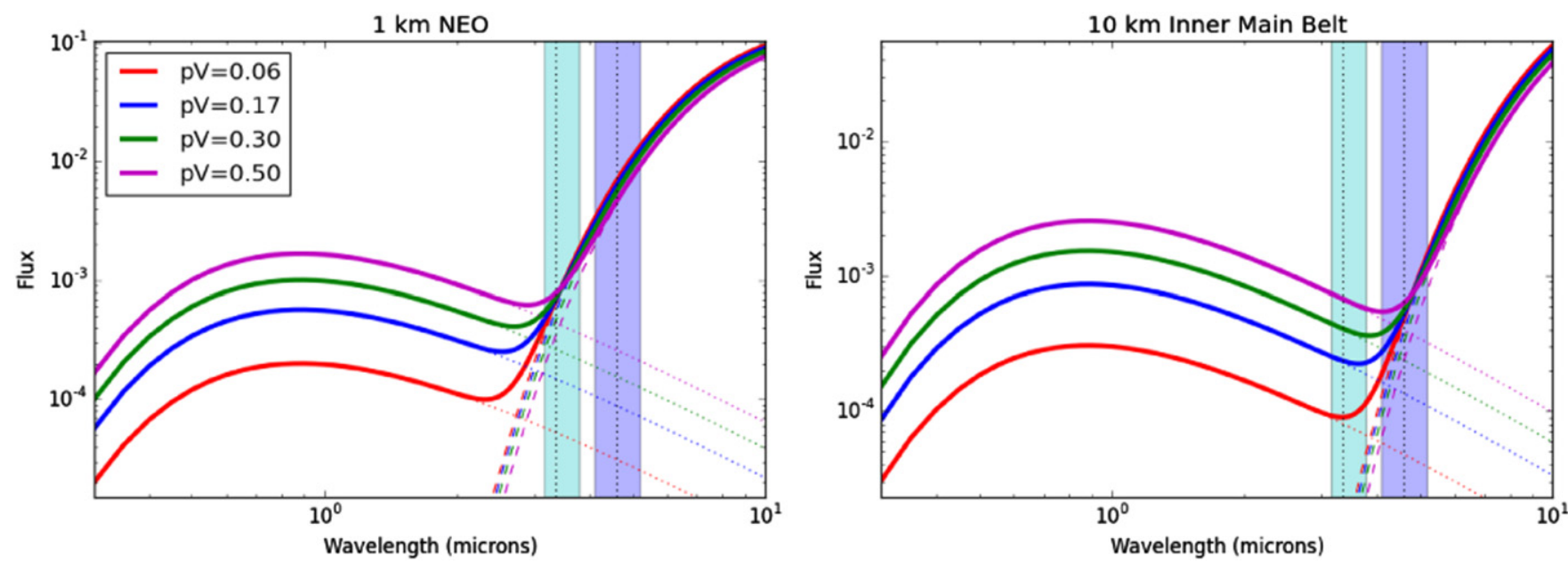

Figure 2. Comparison of spectral energy distribution for a simulated NEO and inner main-belt asteroid, each with albedos ranging from $p_{V}=0.06$ to $p_{V}=0.5$. Thick lines show the flux from the asteroid as a function of wavelength, which is composed of both thermally emitted (dashed) and reflected sunlight (dotted) components. NEOWISE bands centered at 3.4 and $4.6 \mu \mathrm{m}$ are shown as shaded cyan and purple bars, respectively. For NEAs (left), the 3.4 and $4.6 \mu \mathrm{m}$ bands are both thermally dominated. For objects in the inner Main Belt (right), the $3.4 \mu \mathrm{m}$ band is dominated by reflected light, and the $4.6 \mu \mathrm{m}$ band is dominated by thermally emitted light, though the ratio between these components varies with albedo.

reduced photometric accuracy. Following the prescription laid out in Cutri et al. (2012) (Section IV.4), we did not consider objects that were brighter than $8.0 \mathrm{mag}$ at $3.4 \mu \mathrm{m}$ and $7.0 \mathrm{mag}$ at $4.6 \mu \mathrm{m}$. The NEA measurements used in this work are given in Table 1.

\subsection{Near-Earth Thermal Model (NEATM)}

We used the NEATM of Harris (1998), following the implementation of Mainzer et al. (2011b, 2012) for NEAs and Masiero et al. $(2011,2012)$ for MBAs and Mars crossers. These results supersede those published in Mainzer et al. (2014). NEATM is a simple but effective method for determining effective spherical diameters and albedos (when corresponding visible light observations are available). This model makes several assumptions, including a spherical, nonrotating body, with a simple temperature distribution:

$$
T(\theta)=T_{\max } \cos ^{1 / 4}(\theta) \text { for } 0 \leqslant \theta \leqslant \pi / 2
$$

where $\theta$ is the angular distance from the sub-solar point. $T_{\max }$ is the subsolar temperature, defined as:

$$
T_{\max }=\left(\frac{(1-A) S}{\eta \epsilon \sigma}\right)^{1 / 4}
$$

where $A$ is the bolometric Bond albedo, $S$ is the solar flux at the asteroid, $\eta$ is termed the beaming parameter, $\epsilon$ is the emissivity, and $\sigma$ is the Stefan-Boltzmann constant. The beaming parameter $\eta$ accounts for any deviation between the actual asteroid and the model. Changes in $\eta$ can account for a host of factors, including non-spherical shapes, the presence of satellites, variations in surface roughness or thermal inertia, uncertainties in emissivity, high rates of spin, changes in surface temperature distributions due to spin pole location, or the imprecise assumption that the object's night-side has zero thermal emission (a factor that is most relevant for objects observed at high phase angles). Some of these factors that are accounted for in the beaming parameter are degenerate. For example, a slow-rotating object will have a heat distribution similar to a faster rotating object that has a lower thermal inertia. For this simple model, beaming accounts for the changes in temperature distribution due to these effects that cannot be otherwise separated.

Observations were divided into apparitions of 10 days, and the NEATM model was fitted to each individual apparition. These shorter apparitions allowed for fits to widely spaced apparitions or, for NEAs, over changing phase angles. Given that the NEOWISE observational cadence generally results in an object being detected over $\sim 1.5$ days, sometimes with an additional epoch of observations 3-6 months later, we chose to divide observations separated by $>10$ days for separate fitting to account for large changes in object distances and viewing geometries.

NEATM spheres were approximated by a faceted polygon with 800 facets. Individual facet temperature was determined following Equation (1), and then color corrected following Wright et al. (2010). Observed thermal flux for each facet was computed, as was flux from reflected sunlight. The integrated flux from the object was determined, accounting for viewing geometry, to produce a model magnitude. A least-squares fitting routine compared modeled to observed magnitudes, and iterated on diameter, albedo, and beaming parameter until a best fit was found.

Geometric optical albedo $p_{V}$ was computed using absolute magnitude $H$ and slope parameter $G$, using values supplied in MPCORB.dat by the MPC. Inaccurate $H$ and $G$ values will result in inaccurate $p_{V}$ fits. Work by Williams (2012) and Pravec et al. (2012) found systematic $H$ offsets that vary as a function of $H$ magnitude in data reported to the MPC. As albedo measurements depend on $H$ and $G$ values, errors in measurement of those values will propagate to derived albedos.

NEATM requires at least one of the NEOWISE wavelengths to be dominated by thermally emitted light. Some outer mainbelt objects observed by NEOWISE were too cold to have thermally dominated emission at 3.4 or $4.6 \mu \mathrm{m}$, and therefore diameters and albedos for those objects are not reported in this paper. The proportion of reflected versus thermally emitted light for NEAs and inner MBAs can be seen in the spectral energy distribution plots shown in Figure 2. The proportion of thermally emitted flux depends on albedo, which means that for colder, outer MBAs it is unclear if a wavelength is thermally 
Table 2

Measured Diameters $(d)$ and albedos $\left(p_{V}\right)$ of Near-Earth Objects Not Previously Characterized Using NEOWISE Data

\begin{tabular}{|c|c|c|c|c|c|c|c|c|c|}
\hline Name & Packed & $H$ & $G$ & $d(\mathrm{~km})$ & $p_{V}$ & $\eta$ & W2 amp. & $n_{W 1}$ & $n_{W 2}$ \\
\hline 1566 & 01566 & 16.90 & 0.15 & $1.03 \pm 0.04$ & $0.29 \pm 0.05$ & $1.40 \pm 0.00$ & 0.24 & 5 & 7 \\
\hline 1580 & 01580 & 14.50 & 0.15 & $8.55 \pm 5.23$ & $0.04 \pm 0.08$ & $1.40 \pm 0.52$ & 0.34 & 0 & 12 \\
\hline 1620 & 01620 & 15.60 & 0.15 & $1.87 \pm 0.05$ & $0.29 \pm 0.04$ & $1.40 \pm 0.00$ & 0.89 & 14 & 14 \\
\hline 1862 & 01862 & 16.25 & 0.09 & $1.40 \pm 0.04$ & $0.29 \pm 0.04$ & $1.40 \pm 0.00$ & 0.41 & 10 & 10 \\
\hline 1862 & 01862 & 16.25 & 0.09 & $1.26 \pm 0.04$ & $0.35 \pm 0.05$ & $1.40 \pm 0.00$ & 0.84 & 10 & 10 \\
\hline 1917 & 01917 & 13.90 & 0.15 & $4.99 \pm 0.14$ & $0.20 \pm 0.03$ & $1.40 \pm 0.00$ & 0.58 & 14 & 14 \\
\hline 1943 & 01943 & 15.75 & 0.15 & $2.34 \pm 0.05$ & $0.16 \pm 0.02$ & $1.40 \pm 0.00$ & 0.22 & 30 & 31 \\
\hline 1943 & 01943 & 15.75 & 0.15 & $2.30 \pm 0.04$ & $0.17 \pm 0.02$ & $1.40 \pm 0.00$ & 0.29 & 171 & 172 \\
\hline 1943 & 01943 & 15.75 & 0.15 & $2.28 \pm 0.05$ & $0.17 \pm 0.03$ & $1.40 \pm 0.00$ & 0.54 & 14 & 15 \\
\hline 2062 & 02062 & 16.80 & 0.15 & $0.80 \pm 0.03$ & $0.52 \pm 0.10$ & $1.40 \pm 0.00$ & 0.82 & 32 & 36 \\
\hline 3288 & 03288 & 15.20 & 0.15 & $2.49 \pm 0.07$ & $0.24 \pm 0.04$ & $1.40 \pm 0.00$ & 1.40 & 11 & 11 \\
\hline 4954 & 04954 & 12.60 & 0.15 & $9.56 \pm 0.24$ & $0.18 \pm 0.03$ & $1.40 \pm 0.00$ & 1.06 & 8 & 8 \\
\hline 5381 & 05381 & 16.50 & 0.15 & $0.91 \pm 0.05$ & $0.54 \pm 0.07$ & $1.40 \pm 0.00$ & 0.49 & 10 & 10 \\
\hline 5381 & 05381 & 16.50 & 0.15 & $0.94 \pm 0.04$ & $0.51 \pm 0.06$ & $1.40 \pm 0.00$ & 0.17 & 13 & 13 \\
\hline 6053 & 06053 & 14.90 & 0.15 & $3.72 \pm 0.08$ & $0.14 \pm 0.02$ & $1.40 \pm 0.00$ & 0.21 & 11 & 11 \\
\hline 7025 & 07025 & 18.30 & 0.15 & $0.50 \pm 0.17$ & $0.34 \pm 0.23$ & $1.40 \pm 0.52$ & 0.58 & 0 & 4 \\
\hline 7889 & 07889 & 15.20 & 0.15 & $1.68 \pm 0.07$ & $0.52 \pm 0.06$ & $1.40 \pm 0.00$ & 0.45 & 8 & 8 \\
\hline 8567 & 08567 & 15.30 & 0.15 & $2.93 \pm 0.07$ & $0.16 \pm 0.03$ & $1.40 \pm 0.00$ & 0.42 & 25 & 26 \\
\hline 13651 & 13651 & 17.60 & 0.15 & $0.56 \pm 0.02$ & $0.51 \pm 0.11$ & $1.40 \pm 0.00$ & 1.22 & 11 & 11 \\
\hline 35107 & 35107 & 16.80 & 0.15 & $0.91 \pm 0.03$ & $0.41 \pm 0.05$ & $1.40 \pm 0.00$ & 0.25 & 10 & 10 \\
\hline 35107 & 35107 & 16.80 & 0.15 & $1.10 \pm 0.28$ & $0.28 \pm 0.16$ & $1.40 \pm 0.37$ & 0.45 & 0 & 14 \\
\hline 39572 & 39572 & 16.50 & 0.15 & $1.55 \pm 0.66$ & $0.18 \pm 0.16$ & $1.40 \pm 0.47$ & 0.42 & 0 & 8 \\
\hline 39796 & 39796 & 15.70 & 0.15 & $2.13 \pm 0.59$ & $0.20 \pm 0.20$ & $1.40 \pm 0.39$ & 0.69 & 0 & 16 \\
\hline 53430 & 53430 & 16.60 & 0.15 & $1.23 \pm 0.32$ & $0.27 \pm 0.15$ & $1.40 \pm 0.37$ & 1.14 & 0 & 5 \\
\hline 54686 & 54686 & 16.50 & 0.15 & $1.35 \pm 0.46$ & $0.24 \pm 0.19$ & $1.40 \pm 0.47$ & 1.02 & 0 & 10 \\
\hline 55532 & 55532 & 16.10 & 0.15 & $1.31 \pm 0.04$ & $0.38 \pm 0.06$ & $1.40 \pm 0.00$ & 0.22 & 6 & 6 \\
\hline 68063 & 68063 & 15.50 & 0.15 & $2.30 \pm 0.07$ & $0.21 \pm 0.04$ & $1.40 \pm 0.00$ & 0.38 & 24 & 24 \\
\hline 68267 & 68267 & 16.90 & 0.15 & $0.88 \pm 0.04$ & $0.40 \pm 0.05$ & $1.40 \pm 0.00$ & 0.29 & 13 & 15 \\
\hline 68348 & 68348 & 14.20 & 0.15 & $3.51 \pm 0.13$ & $0.30 \pm 0.05$ & $1.40 \pm 0.00$ & 0.46 & 12 & 12 \\
\hline 68548 & 68548 & 16.50 & 0.15 & $1.18 \pm 0.04$ & $0.32 \pm 0.04$ & $1.40 \pm 0.00$ & 0.24 & 8 & 10 \\
\hline 68548 & 68548 & 16.50 & 0.15 & $1.24 \pm 0.04$ & $0.29 \pm 0.03$ & $1.40 \pm 0.00$ & 0.55 & 23 & 24 \\
\hline 85182 & 85182 & 17.10 & 0.15 & $1.03 \pm 0.37$ & $0.24 \pm 0.19$ & $1.40 \pm 0.49$ & 0.69 & 0 & 9 \\
\hline 85774 & 85774 & 19.20 & 0.15 & $0.94 \pm 0.01$ & $0.04 \pm 0.01$ & $1.40 \pm 0.00$ & 0.90 & 11 & 11 \\
\hline 86819 & 86819 & 17.40 & 0.15 & $0.80 \pm 0.27$ & $0.30 \pm 0.22$ & $1.40 \pm 0.46$ & 0.79 & 0 & 7 \\
\hline 86829 & 86829 & 15.90 & 0.15 & $1.43 \pm 0.05$ & $0.37 \pm 0.05$ & $1.40 \pm 0.00$ & 0.33 & 14 & 14 \\
\hline 87309 & 87309 & 17.60 & 0.15 & $0.57 \pm 0.16$ & $0.50 \pm 0.23$ & $1.40 \pm 0.47$ & 0.67 & 0 & 10 \\
\hline 88213 & 88213 & 19.70 & 0.15 & $0.91 \pm 0.42$ & $0.03 \pm 0.03$ & $1.40 \pm 0.51$ & 0.66 & 0 & 6 \\
\hline 89355 & 89355 & 15.60 & 0.15 & $2.04 \pm 0.05$ & $0.25 \pm 0.03$ & $1.40 \pm 0.00$ & 1.19 & 30 & 31 \\
\hline 90075 & 90075 & 15.20 & 0.15 & $2.23 \pm 0.08$ & $0.29 \pm 0.04$ & $1.40 \pm 0.00$ & 0.73 & 12 & 12 \\
\hline 99248 & 99248 & 16.30 & 0.15 & $1.12 \pm 0.04$ & $0.43 \pm 0.06$ & $1.40 \pm 0.00$ & 0.29 & 7 & 8 \\
\hline 99248 & 99248 & 16.30 & 0.15 & $1.14 \pm 0.37$ & $0.41 \pm 0.28$ & $1.40 \pm 0.48$ & 0.48 & 0 & 8 \\
\hline 137099 & D7099 & 18.20 & 0.15 & $0.56 \pm 0.02$ & $0.29 \pm 0.04$ & $1.40 \pm 0.00$ & 0.65 & 6 & 6 \\
\hline 138127 & D8127 & 17.10 & 0.15 & $0.75 \pm 0.02$ & $0.45 \pm 0.06$ & $1.40 \pm 0.00$ & 0.17 & 7 & 7 \\
\hline 138947 & D8947 & 18.70 & 0.15 & $0.45 \pm 0.12$ & $0.29 \pm 0.28$ & $1.40 \pm 0.46$ & 0.46 & 0 & 9 \\
\hline 142781 & E2781 & 16.10 & 0.15 & $1.59 \pm 0.05$ & $0.25 \pm 0.04$ & $1.40 \pm 0.00$ & 0.15 & 14 & 14 \\
\hline 142781 & E2781 & 16.10 & 0.15 & $2.01 \pm 0.74$ & $0.16 \pm 0.15$ & $1.40 \pm 0.44$ & 0.85 & 0 & 15 \\
\hline 142781 & E2781 & 16.10 & 0.15 & $2.03 \pm 0.77$ & $0.16 \pm 0.09$ & $1.40 \pm 0.40$ & 0.45 & 0 & 9 \\
\hline 143624 & E3624 & 15.90 & 0.15 & $2.14 \pm 0.04$ & $0.17 \pm 0.03$ & $1.40 \pm 0.00$ & 0.32 & 9 & 9 \\
\hline 143624 & E3624 & 15.90 & 0.15 & $2.23 \pm 1.08$ & $0.15 \pm 0.17$ & $1.40 \pm 0.53$ & 0.82 & 0 & 8 \\
\hline 154276 & F4276 & 17.60 & 0.15 & $1.06 \pm 0.35$ & $0.14 \pm 0.17$ & $1.40 \pm 0.43$ & 0.29 & 0 & 5 \\
\hline 159454 & F9454 & 17.90 & 0.15 & $0.58 \pm 0.02$ & $0.37 \pm 0.04$ & $1.40 \pm 0.00$ & 0.30 & 6 & 6 \\
\hline 159560 & F9560 & 17.00 & 0.15 & $1.10 \pm 0.47$ & $0.24 \pm 0.23$ & $1.40 \pm 0.54$ & 1.16 & 0 & 87 \\
\hline 159560 & F9560 & 17.00 & 0.15 & $1.16 \pm 0.30$ & $0.21 \pm 0.21$ & $1.40 \pm 0.39$ & 0.53 & 0 & 13 \\
\hline 159857 & F9857 & 15.40 & 0.15 & $3.07 \pm 1.32$ & $0.13 \pm 0.16$ & $1.40 \pm 0.45$ & 0.34 & 0 & 5 \\
\hline 162058 & G2058 & 17.80 & 0.15 & $0.85 \pm 0.01$ & $0.19 \pm 0.02$ & $1.40 \pm 0.00$ & 0.34 & 26 & 27 \\
\hline 162058 & G2058 & 17.80 & 0.15 & $0.85 \pm 0.28$ & $0.19 \pm 0.14$ & $1.40 \pm 0.44$ & 0.87 & 0 & 31 \\
\hline 162080 & G2080 & 19.80 & 0.15 & $0.78 \pm 0.06$ & $0.04 \pm 0.01$ & $1.40 \pm 0.11$ & 1.39 & 4 & 4 \\
\hline 162080 & G2080 & 19.80 & 0.15 & $0.82 \pm 0.33$ & $0.03 \pm 0.07$ & $1.40 \pm 0.49$ & 0.99 & 0 & 13 \\
\hline 162116 & G2116 & 19.30 & 0.15 & $0.54 \pm 0.17$ & $0.12 \pm 0.08$ & $1.40 \pm 0.40$ & 0.47 & 0 & 7 \\
\hline 162567 & G2567 & 19.90 & 0.15 & $0.33 \pm 0.01$ & $0.17 \pm 0.03$ & $1.40 \pm 0.00$ & 0.20 & 6 & 6 \\
\hline 162741 & G2741 & 17.30 & 0.15 & $3.95 \pm 0.04$ & $0.01 \pm 0.00$ & $1.40 \pm 0.00$ & 0.22 & 6 & 6 \\
\hline 162980 & G2980 & 16.70 & 0.15 & $0.79 \pm 0.04$ & $0.66 \pm 0.13$ & $1.40 \pm 0.00$ & 0.40 & 8 & 8 \\
\hline 163818 & G3818 & 18.40 & 0.15 & $0.39 \pm 0.02$ & $0.52 \pm 0.06$ & $1.40 \pm 0.00$ & 0.33 & 7 & 7 \\
\hline 172034 & H2034 & 17.80 & 0.15 & $0.63 \pm 0.02$ & $0.34 \pm 0.05$ & $1.40 \pm 0.00$ & 1.05 & 16 & 16 \\
\hline 190166 & J0166 & 17.10 & 0.15 & $1.01 \pm 0.03$ & $0.25 \pm 0.04$ & $1.40 \pm 0.00$ & 0.92 & 6 & 7 \\
\hline
\end{tabular}


Table 2

(Continued)

\begin{tabular}{|c|c|c|c|c|c|c|c|c|c|}
\hline Name & Packed & $H$ & $G$ & $d(\mathrm{~km})$ & $p_{V}$ & $\eta$ & W2 amp. & $n_{W 1}$ & $n_{W 2}$ \\
\hline 190166 & J0166 & 17.10 & 0.15 & $1.05 \pm 0.02$ & $0.23 \pm 0.03$ & $1.40 \pm 0.00$ & 0.68 & 12 & 12 \\
\hline 209924 & K9924 & 16.10 & 0.15 & $1.86 \pm 0.71$ & $0.19 \pm 0.12$ & $1.40 \pm 0.44$ & 0.40 & 0 & 7 \\
\hline 211871 & L1871 & 18.80 & 0.15 & $0.41 \pm 0.01$ & $0.32 \pm 0.05$ & $1.40 \pm 0.00$ & 0.28 & 5 & 7 \\
\hline 214088 & L4088 & 15.20 & 0.15 & $2.42 \pm 0.06$ & $0.25 \pm 0.03$ & $1.40 \pm 0.00$ & 0.63 & 8 & 8 \\
\hline 215588 & L5588 & 19.50 & 0.15 & $0.49 \pm 0.16$ & $0.12 \pm 0.12$ & $1.40 \pm 0.44$ & 0.57 & 0 & 5 \\
\hline 215757 & L5757 & 17.70 & 0.15 & $0.78 \pm 0.27$ & $0.24 \pm 0.17$ & $1.40 \pm 0.48$ & 0.47 & 0 & 11 \\
\hline 235086 & N5086 & 17.50 & 0.15 & $1.02 \pm 0.40$ & $0.17 \pm 0.18$ & $1.40 \pm 0.51$ & 1.04 & 0 & 60 \\
\hline 235086 & N5086 & 17.50 & 0.15 & $1.02 \pm 0.32$ & $0.17 \pm 0.11$ & $1.40 \pm 0.38$ & 1.64 & 0 & 32 \\
\hline 235086 & N5086 & 17.50 & 0.15 & $1.08 \pm 0.33$ & $0.15 \pm 0.12$ & $1.40 \pm 0.38$ & 0.85 & 0 & 29 \\
\hline 242450 & $\mathrm{O} 2450$ & 14.70 & 0.15 & $2.54 \pm 0.10$ & $0.36 \pm 0.13$ & $1.40 \pm 0.00$ & 0.41 & 11 & 11 \\
\hline 242450 & $\mathrm{O} 2450$ & 14.70 & 0.15 & $2.91 \pm 0.08$ & $0.27 \pm 0.04$ & $1.40 \pm 0.00$ & 0.83 & 13 & 14 \\
\hline 250620 & P0620 & 18.00 & 0.15 & $0.65 \pm 0.14$ & $0.26 \pm 0.13$ & $1.40 \pm 0.33$ & 0.29 & 0 & 4 \\
\hline 267337 & Q7337 & 18.00 & 0.15 & $0.44 \pm 0.10$ & $0.58 \pm 0.25$ & $1.40 \pm 0.43$ & 0.21 & 0 & 4 \\
\hline 269690 & Q9690 & 18.40 & 0.15 & $0.89 \pm 0.43$ & $0.10 \pm 0.11$ & $1.40 \pm 0.59$ & 0.31 & 0 & 7 \\
\hline 271480 & $\mathrm{R} 1480$ & 17.50 & 0.15 & $0.71 \pm 0.22$ & $0.35 \pm 0.22$ & $1.40 \pm 0.48$ & 0.82 & 0 & 6 \\
\hline 274138 & R4138 & 17.80 & 0.15 & $0.75 \pm 0.02$ & $0.24 \pm 0.03$ & $1.40 \pm 0.00$ & 0.48 & 7 & 7 \\
\hline 275976 & R5976 & 16.30 & 0.15 & $1.86 \pm 0.04$ & $0.15 \pm 0.03$ & $1.40 \pm 0.00$ & 1.01 & 5 & 5 \\
\hline 275976 & R5976 & 16.30 & 0.15 & $2.38 \pm 0.03$ & $0.09 \pm 0.01$ & $1.40 \pm 0.00$ & 1.11 & 15 & 16 \\
\hline 276274 & R6274 & 17.20 & 0.15 & $1.53 \pm 0.71$ & $0.10 \pm 0.17$ & $1.40 \pm 0.52$ & 0.91 & 0 & 5 \\
\hline 276468 & R6468 & 17.90 & 0.15 & $1.03 \pm 0.37$ & $0.11 \pm 0.14$ & $1.40 \pm 0.42$ & 0.36 & 0 & 5 \\
\hline 285944 & S5944 & 16.50 & 0.15 & $1.04 \pm 0.04$ & $0.41 \pm 0.03$ & $1.40 \pm 0.00$ & 0.16 & 10 & 10 \\
\hline 285944 & S5944 & 16.50 & 0.15 & $1.40 \pm 0.43$ & $0.23 \pm 0.19$ & $1.40 \pm 0.41$ & 0.51 & 0 & 29 \\
\hline 297418 & T7418 & 18.60 & 0.15 & $0.41 \pm 0.02$ & $0.39 \pm 0.05$ & $1.40 \pm 0.00$ & 0.93 & 5 & 5 \\
\hline 299582 & T9582 & 18.00 & 0.15 & $0.62 \pm 0.02$ & $0.29 \pm 0.03$ & $1.40 \pm 0.00$ & 0.31 & 7 & 7 \\
\hline 303174 & U3174 & 16.70 & 0.15 & $1.50 \pm 0.03$ & $0.16 \pm 0.03$ & $1.40 \pm 0.00$ & 0.65 & 21 & 23 \\
\hline 304330 & U4330 & 18.90 & 0.15 & $0.61 \pm 0.01$ & $0.13 \pm 0.02$ & $1.40 \pm 0.00$ & 0.13 & 11 & 11 \\
\hline 304330 & U4330 & 18.90 & 0.15 & $0.78 \pm 0.01$ & $0.08 \pm 0.01$ & $1.40 \pm 0.00$ & 0.23 & 12 & 12 \\
\hline 322763 & W2763 & 16.90 & 0.15 & $1.25 \pm 0.03$ & $0.20 \pm 0.04$ & $1.40 \pm 0.00$ & 0.27 & 12 & 13 \\
\hline 326388 & W6388 & 18.20 & 0.15 & $1.26 \pm 0.57$ & $0.06 \pm 0.12$ & $1.40 \pm 0.52$ & 0.33 & 0 & 8 \\
\hline 334673 & X4673 & 17.90 & 0.15 & $0.57 \pm 0.22$ & $0.38 \pm 0.25$ & $1.40 \pm 0.60$ & 0.67 & 0 & 11 \\
\hline 349219 & Y9219 & 18.20 & 0.15 & $0.58 \pm 0.15$ & $0.27 \pm 0.23$ & $1.40 \pm 0.41$ & 0.58 & 0 & 14 \\
\hline 363505 & a3505 & 18.10 & 0.15 & $1.90 \pm 0.05$ & $0.03 \pm 0.01$ & $1.40 \pm 0.03$ & 0.66 & 12 & 12 \\
\hline 368184 & a8184 & 19.50 & 0.15 & $0.38 \pm 0.12$ & $0.19 \pm 0.19$ & $1.40 \pm 0.46$ & 0.54 & 0 & 25 \\
\hline 369264 & a9264 & 16.30 & 0.15 & $1.51 \pm 0.47$ & $0.23 \pm 0.20$ & $1.40 \pm 0.42$ & 0.65 & 0 & 7 \\
\hline 377732 & b7732 & 17.00 & 0.15 & $0.95 \pm 0.03$ & $0.31 \pm 0.05$ & $1.40 \pm 0.00$ & 0.62 & 5 & 5 \\
\hline 377732 & b7732 & 17.00 & 0.15 & $0.99 \pm 0.03$ & $0.29 \pm 0.03$ & $1.40 \pm 0.00$ & 0.16 & 5 & 5 \\
\hline 381677 & c1677 & 18.40 & 0.15 & $0.47 \pm 0.01$ & $0.35 \pm 0.05$ & $1.40 \pm 0.00$ & 0.92 & 19 & 19 \\
\hline 381677 & c1677 & 18.40 & 0.15 & $0.44 \pm 0.16$ & $0.40 \pm 0.21$ & $1.40 \pm 0.54$ & 0.47 & 0 & 5 \\
\hline 387733 & c7733 & 18.90 & 0.15 & $0.34 \pm 0.01$ & $0.41 \pm 0.06$ & $1.40 \pm 0.00$ & 0.22 & 11 & 11 \\
\hline 387733 & c7733 & 18.90 & 0.15 & $0.32 \pm 0.09$ & $0.47 \pm 0.25$ & $1.40 \pm 0.46$ & 0.37 & 0 & 5 \\
\hline 387746 & c7746 & 20.00 & 0.15 & $0.37 \pm 0.01$ & $0.13 \pm 0.02$ & $1.40 \pm 0.00$ & 0.23 & 5 & 6 \\
\hline 388838 & c8838 & 19.50 & 0.15 & $0.36 \pm 0.01$ & $0.21 \pm 0.04$ & $1.40 \pm 0.00$ & 0.61 & 18 & 18 \\
\hline 388838 & c8838 & 19.50 & 0.15 & $0.38 \pm 0.01$ & $0.20 \pm 0.02$ & $1.40 \pm 0.00$ & 0.24 & 12 & 12 \\
\hline 389694 & c9694 & 18.20 & 0.15 & $0.45 \pm 0.02$ & $0.46 \pm 0.06$ & $1.40 \pm 0.00$ & 0.22 & 4 & 5 \\
\hline 391211 & $\mathrm{~d} 1211$ & 18.50 & 0.15 & $0.41 \pm 0.09$ & $0.42 \pm 0.23$ & $1.40 \pm 0.38$ & 0.85 & 0 & 17 \\
\hline 393359 & d3359 & 19.20 & 0.15 & $0.77 \pm 0.33$ & $0.06 \pm 0.11$ & $1.40 \pm 0.52$ & 0.51 & 0 & 30 \\
\hline 393569 & d3569 & 20.20 & 0.15 & $0.55 \pm 0.01$ & $0.05 \pm 0.01$ & $1.40 \pm 0.00$ & 0.22 & 13 & 14 \\
\hline 399433 & d9433 & 18.60 & 0.15 & $1.34 \pm 0.56$ & $0.04 \pm 0.09$ & $1.40 \pm 0.49$ & 0.24 & 0 & 10 \\
\hline 399433 & d9433 & 18.60 & 0.15 & $1.76 \pm 0.89$ & $0.02 \pm 0.05$ & $1.40 \pm 0.53$ & 0.16 & 0 & 9 \\
\hline 406952 & e6952 & 17.10 & 0.15 & $0.77 \pm 0.21$ & $0.43 \pm 0.23$ & $1.40 \pm 0.44$ & 0.64 & 0 & 5 \\
\hline 408751 & e8751 & 19.00 & 0.15 & $0.40 \pm 0.01$ & $0.28 \pm 0.03$ & $1.40 \pm 0.00$ & 0.84 & 68 & 69 \\
\hline 409256 & e9256 & 18.20 & 0.15 & $1.89 \pm 0.68$ & $0.03 \pm 0.04$ & $1.40 \pm 0.40$ & 0.84 & 0 & 4 \\
\hline 409836 & e9836 & 18.10 & 0.15 & $0.55 \pm 0.19$ & $0.33 \pm 0.25$ & $1.40 \pm 0.49$ & 1.78 & 0 & 14 \\
\hline 410088 & f0088 & 18.10 & 0.15 & $1.03 \pm 0.01$ & $0.10 \pm 0.02$ & $1.40 \pm 0.00$ & 0.14 & 9 & 10 \\
\hline 410778 & f0778 & 18.10 & 0.15 & $1.46 \pm 0.57$ & $0.05 \pm 0.03$ & $1.40 \pm 0.41$ & 0.38 & 0 & 6 \\
\hline 411201 & f1201 & 17.80 & 0.15 & $0.66 \pm 0.01$ & $0.31 \pm 0.05$ & $1.40 \pm 0.00$ & 1.45 & 12 & 15 \\
\hline 411611 & f1611 & 18.80 & 0.15 & $0.36 \pm 0.10$ & $0.41 \pm 0.21$ & $1.40 \pm 0.43$ & 0.81 & 0 & 31 \\
\hline 413038 & f 3038 & 16.90 & 0.15 & $1.24 \pm 0.03$ & $0.20 \pm 0.04$ & $1.40 \pm 0.00$ & 1.19 & 22 & 23 \\
\hline 413038 & f3038 & 16.90 & 0.15 & $1.01 \pm 0.04$ & $0.30 \pm 0.04$ & $1.40 \pm 0.00$ & 1.79 & 23 & 25 \\
\hline 413192 & f3192 & 16.80 & 0.15 & $3.96 \pm 1.84$ & $0.02 \pm 0.05$ & $1.40 \pm 0.47$ & 0.62 & 0 & 18 \\
\hline 413421 & f 3421 & 18.30 & 0.15 & $1.90 \pm 0.78$ & $0.02 \pm 0.02$ & $1.40 \pm 0.41$ & 1.39 & 0 & 23 \\
\hline 413820 & f 3820 & 19.80 & 0.15 & $0.66 \pm 0.26$ & $0.05 \pm 0.04$ & $1.40 \pm 0.46$ & 0.97 & 0 & 36 \\
\hline 414286 & $\mathrm{f} 4286$ & 18.60 & 0.15 & $0.37 \pm 0.08$ & $0.47 \pm 0.19$ & $1.40 \pm 0.38$ & 0.54 & 0 & 27 \\
\hline 414286 & $\mathrm{f} 4286$ & 18.60 & 0.15 & $0.40 \pm 0.09$ & $0.40 \pm 0.24$ & $1.40 \pm 0.40$ & 0.71 & 0 & 29 \\
\hline
\end{tabular}


Table 2

(Continued)

\begin{tabular}{|c|c|c|c|c|c|c|c|c|c|}
\hline Name & Packed & $H$ & $G$ & $d(\mathrm{~km})$ & $p_{V}$ & $\eta$ & W2 amp. & $n_{W 1}$ & $n_{W 2}$ \\
\hline 418797 & f8797 & 19.40 & 0.15 & $0.70 \pm 0.29$ & $0.06 \pm 0.07$ & $1.40 \pm 0.50$ & 0.32 & 0 & 7 \\
\hline 418929 & f8929 & 17.00 & 0.15 & $1.43 \pm 0.02$ & $0.14 \pm 0.03$ & $1.40 \pm 0.00$ & 0.54 & 48 & 49 \\
\hline 419624 & f9624 & 20.50 & 0.15 & $0.34 \pm 0.14$ & $0.09 \pm 0.17$ & $1.40 \pm 0.50$ & 0.57 & 0 & 18 \\
\hline 419624 & f9624 & 20.50 & 0.15 & $0.36 \pm 0.13$ & $0.09 \pm 0.14$ & $1.40 \pm 0.46$ & 0.46 & 0 & 6 \\
\hline 419880 & f9880 & 19.60 & 0.15 & $0.98 \pm 0.06$ & $0.03 \pm 0.01$ & $1.40 \pm 0.08$ & 0.20 & 6 & 6 \\
\hline 2000 AG205 & K00AK5G & 19.70 & 0.15 & $0.95 \pm 0.01$ & $0.03 \pm 0.00$ & $1.40 \pm 0.00$ & 0.79 & 12 & 14 \\
\hline 2002 XS40 & K02X40S & 20.10 & 0.15 & $0.76 \pm 0.03$ & $0.03 \pm 0.00$ & $1.40 \pm 0.05$ & 0.18 & 14 & 14 \\
\hline 2003 CC11 & $\mathrm{K} 03 \mathrm{C} 11 \mathrm{C}$ & 19.10 & 0.15 & $1.13 \pm 0.51$ & $0.03 \pm 0.10$ & $1.40 \pm 0.53$ & 0.49 & 0 & 16 \\
\hline 2003 SS214 & K03SL4S & 20.10 & 0.15 & $0.86 \pm 0.25$ & $0.02 \pm 0.02$ & $1.40 \pm 0.35$ & 0.75 & 0 & 16 \\
\hline 2004 BZ74 & K04B74Z & 18.10 & 0.15 & $0.96 \pm 0.02$ & $0.11 \pm 0.02$ & $1.40 \pm 0.00$ & 0.65 & 4 & 5 \\
\hline 2004 MX2 & K04M02X & 19.30 & 0.15 & $1.26 \pm 0.08$ & $0.02 \pm 0.00$ & $1.40 \pm 0.09$ & 0.35 & 9 & 9 \\
\hline 2004 TG10 & K04T10G & 19.40 & 0.15 & $1.32 \pm 0.61$ & $0.02 \pm 0.04$ & $1.40 \pm 0.51$ & 0.64 & 0 & 8 \\
\hline 2005 LS3 & K05L03S & 19.50 & 0.15 & $0.38 \pm 0.10$ & $0.19 \pm 0.12$ & $1.40 \pm 0.38$ & 0.64 & 0 & 7 \\
\hline 2006 BB27 & К06B27B & 20.00 & 0.15 & $0.22 \pm 0.05$ & $0.38 \pm 0.21$ & $1.40 \pm 0.38$ & 0.98 & 0 & 5 \\
\hline 2007 BG & K07B00G & 19.50 & 0.15 & $0.31 \pm 0.11$ & $0.24 \pm 0.19$ & $1.40 \pm 0.51$ & 0.38 & 3 & 5 \\
\hline 2007 RU10 & K07R10U & 19.10 & 0.15 & $0.92 \pm 0.37$ & $0.05 \pm 0.06$ & $1.40 \pm 0.47$ & 0.31 & 0 & 9 \\
\hline 2008 QS11 & K08Q11S & 19.90 & 0.15 & $0.45 \pm 0.01$ & $0.09 \pm 0.01$ & $1.40 \pm 0.00$ & 0.33 & 9 & 11 \\
\hline 2009 ND1 & K09N01D & 17.10 & 0.15 & $2.50 \pm 0.95$ & $0.04 \pm 0.04$ & $1.40 \pm 0.39$ & 0.70 & 0 & 11 \\
\hline 2010 OQ1 & K10O01Q & 19.00 & 0.15 & $0.54 \pm 0.21$ & $0.15 \pm 0.14$ & $1.40 \pm 0.51$ & 0.47 & 0 & 8 \\
\hline $2011 \mathrm{CQ} 4$ & $\mathrm{~K} 11 \mathrm{C} 04 \mathrm{Q}$ & 18.40 & 0.15 & $0.66 \pm 0.02$ & $0.18 \pm 0.02$ & $1.40 \pm 0.00$ & 0.29 & 5 & 7 \\
\hline 2012 DN & K12D00N & 18.10 & 0.15 & $2.77 \pm 1.05$ & $0.01 \pm 0.03$ & $1.40 \pm 0.38$ & 0.42 & 0 & 7 \\
\hline 2013 PX6 & K13P06X & 18.40 & 0.15 & $1.65 \pm 0.03$ & $0.03 \pm 0.00$ & $1.40 \pm 0.02$ & 0.19 & 9 & 10 \\
\hline 2013 WT44 & K13W44T & 19.30 & 0.15 & $0.65 \pm 0.01$ & $0.08 \pm 0.02$ & $1.40 \pm 0.00$ & 0.31 & 6 & 6 \\
\hline 2013 WU44 & K13W44U & 21.00 & 0.15 & $0.29 \pm 0.13$ & $0.09 \pm 0.19$ & $1.40 \pm 0.61$ & 0.26 & 0 & 8 \\
\hline 2013 YZ13 & K13Y13Z & 19.60 & 0.15 & $0.31 \pm 0.10$ & $0.27 \pm 0.19$ & $1.40 \pm 0.46$ & 0.07 & 0 & 6 \\
\hline 2013 YP139 & K13YD9P & 21.60 & 0.15 & $0.40 \pm 0.03$ & $0.03 \pm 0.01$ & $1.09 \pm 0.07$ & 0.25 & 6 & 6 \\
\hline 2014 AA33 & K14A33A & 19.30 & 0.15 & $0.79 \pm 0.04$ & $0.05 \pm 0.01$ & $1.40 \pm 0.06$ & 0.19 & 4 & 4 \\
\hline 2014 AQ46 & K14A46Q & 20.10 & 0.15 & $0.59 \pm 0.29$ & $0.05 \pm 0.11$ & $1.40 \pm 0.60$ & 0.47 & 0 & 17 \\
\hline 2014 AA53 & K14A53A & 19.80 & 0.15 & $0.70 \pm 0.27$ & $0.04 \pm 0.06$ & $1.40 \pm 0.47$ & 0.50 & 0 & 13 \\
\hline 2014 BG60 & K14B60G & 20.10 & 0.15 & $0.67 \pm 0.25$ & $0.04 \pm 0.08$ & $1.40 \pm 0.46$ & 1.30 & 0 & 163 \\
\hline 2014 BE63 & K14B63E & 23.20 & 0.15 & $0.36 \pm 0.13$ & $0.01 \pm 0.00$ & $1.40 \pm 0.46$ & 0.42 & 0 & 5 \\
\hline 2014 CY4 & $\mathrm{K} 14 \mathrm{C} 04 \mathrm{Y}$ & 21.10 & 0.15 & $0.57 \pm 0.25$ & $0.02 \pm 0.04$ & $1.40 \pm 0.52$ & 0.35 & 0 & 5 \\
\hline 2014 DC10 & K14D10C & 20.10 & 0.15 & $0.89 \pm 0.01$ & $0.02 \pm 0.00$ & $1.40 \pm 0.00$ & 0.90 & 9 & 10 \\
\hline 2014 ED & K14E00D & 19.30 & 0.15 & $0.49 \pm 0.13$ & $0.14 \pm 0.14$ & $1.40 \pm 0.39$ & 0.57 & 0 & 6 \\
\hline 2014 EN45 & $\mathrm{K} 14 \mathrm{E} 45 \mathrm{~N}$ & 21.20 & 0.15 & $0.37 \pm 0.13$ & $0.04 \pm 0.01$ & $0.75 \pm 0.24$ & 0.16 & 12 & 12 \\
\hline 2014 EZ48 & K14E48Z & 18.80 & 0.15 & $0.45 \pm 0.01$ & $0.26 \pm 0.04$ & $1.40 \pm 0.00$ & 1.10 & 5 & 6 \\
\hline 2014 EZ48 & K14E48Z & 18.80 & 0.15 & $0.44 \pm 0.11$ & $0.27 \pm 0.21$ & $1.40 \pm 0.38$ & 0.47 & 0 & 6 \\
\hline 2014 EQ49 & K14E49Q & 21.80 & 0.15 & $0.38 \pm 0.13$ & $0.02 \pm 0.03$ & $1.40 \pm 0.42$ & 0.42 & 0 & 5 \\
\hline 2014 ER49 & K14E49R & 18.60 & 0.15 & $0.46 \pm 0.15$ & $0.30 \pm 0.26$ & $1.40 \pm 0.49$ & 0.51 & 0 & 9 \\
\hline 2014 HE3 & K14H03E & 19.90 & 0.15 & $0.56 \pm 0.15$ & $0.06 \pm 0.04$ & $1.40 \pm 0.34$ & 0.18 & 0 & 5 \\
\hline 2014 HQ124 & K14HC4Q & 18.90 & 0.15 & $0.41 \pm 0.17$ & $0.29 \pm 0.22$ & $1.40 \pm 0.57$ & 0.80 & 0 & 10 \\
\hline 2014 HF177 & K14HH7F & 19.70 & 0.15 & $0.25 \pm 0.01$ & $0.36 \pm 0.06$ & $1.40 \pm 0.00$ & 0.39 & 10 & 12 \\
\hline 2014 JL25 & K14J25L & 23.00 & 0.15 & $0.23 \pm 0.06$ & $0.02 \pm 0.03$ & $1.40 \pm 0.34$ & 0.68 & 0 & 5 \\
\hline 2014 JH57 & K14J57H & 16.60 & 0.15 & $4.61 \pm 0.03$ & $0.02 \pm 0.00$ & $1.40 \pm 0.00$ & 0.11 & 6 & 6 \\
\hline 2014 JH57 & K14J57H & 16.60 & 0.15 & $6.79 \pm 3.81$ & $0.01 \pm 0.03$ & $1.40 \pm 0.47$ & 0.30 & 0 & 5 \\
\hline 2014 JN57 & K14J57N & 20.70 & 0.15 & $0.27 \pm 0.10$ & $0.12 \pm 0.10$ & $1.40 \pm 0.47$ & 0.69 & 0 & 4 \\
\hline 2014 KX99 & K14K99X & 18.20 & 0.15 & $1.72 \pm 0.68$ & $0.03 \pm 0.05$ & $1.40 \pm 0.46$ & 0.43 & 0 & 9 \\
\hline 2014 LQ25 & K14L25Q & 20.00 & 0.15 & $0.94 \pm 0.32$ & $0.02 \pm 0.01$ & $1.40 \pm 0.37$ & 0.48 & 0 & 5 \\
\hline 2014 LR26 & K14L26R & 18.50 & 0.15 & $2.08 \pm 0.90$ & $0.02 \pm 0.03$ & $1.40 \pm 0.46$ & 0.65 & 0 & 6 \\
\hline 2014 MQ18 & K14M18Q & 15.60 & 0.15 & $5.27 \pm 3.50$ & $0.04 \pm 0.07$ & $1.40 \pm 0.52$ & 0.54 & 0 & 8 \\
\hline 2014 NB39 & K14N39B & 19.50 & 0.15 & $1.08 \pm 0.15$ & $0.02 \pm 0.02$ & $1.40 \pm 0.18$ & 0.08 & 7 & 7 \\
\hline 2014 NE52 & K14N52E & 17.90 & 0.15 & $0.70 \pm 0.22$ & $0.25 \pm 0.27$ & $1.40 \pm 0.47$ & 0.66 & 0 & 9 \\
\hline 2014 NC64 & K14N64C & 20.50 & 0.15 & $0.50 \pm 0.19$ & $0.04 \pm 0.02$ & $0.82 \pm 0.29$ & 0.64 & 5 & 6 \\
\hline 2014 NM64 & K14N64M & 22.60 & 0.15 & $0.33 \pm 0.12$ & $0.01 \pm 0.02$ & $1.40 \pm 0.44$ & 0.82 & 0 & 25 \\
\hline 2014 OY1 & K14O01Y & 19.10 & 0.15 & $0.60 \pm 0.21$ & $0.11 \pm 0.09$ & $1.40 \pm 0.43$ & 0.30 & 0 & 6 \\
\hline 2014 OZ1 & K14O01Z & 21.00 & 0.15 & $0.73 \pm 0.29$ & $0.01 \pm 0.03$ & $1.40 \pm 0.49$ & 0.38 & 0 & 21 \\
\hline 2014 PC68 & K14P68C & 20.40 & 0.15 & $0.56 \pm 0.20$ & $0.04 \pm 0.04$ & $1.40 \pm 0.43$ & 0.39 & 0 & 8 \\
\hline 2014 PF68 & K14P68F & 18.20 & 0.15 & $3.33 \pm 2.06$ & $0.01 \pm 0.01$ & $1.20 \pm 0.48$ & 0.60 & 0 & 12 \\
\hline 2014 QK433 & K14Qh3K & 18.30 & 0.15 & $1.78 \pm 0.75$ & $0.03 \pm 0.06$ & $1.40 \pm 0.47$ & 0.79 & 0 & 10 \\
\hline 2014 RH12 & $\mathrm{K} 14 \mathrm{R} 12 \mathrm{H}$ & 23.50 & 0.15 & $0.09 \pm 0.04$ & $0.09 \pm 0.11$ & $1.40 \pm 0.54$ & 0.75 & 0 & 10 \\
\hline 2014 RL12 & K14R12L & 17.90 & 0.15 & $0.69 \pm 0.02$ & $0.25 \pm 0.03$ & $1.40 \pm 0.00$ & 0.31 & 5 & 5 \\
\hline 2014 RL12 & K14R12L & 17.90 & 0.15 & $0.61 \pm 0.17$ & $0.33 \pm 0.19$ & $1.40 \pm 0.42$ & 0.83 & 0 & 6 \\
\hline 2014 SR339 & K14SX9R & 18.60 & 0.15 & $0.97 \pm 0.37$ & $0.07 \pm 0.07$ & $1.40 \pm 0.46$ & 0.69 & 0 & 13 \\
\hline 2014 TW57 & K14T57W & 20.10 & 0.15 & $0.47 \pm 0.01$ & $0.07 \pm 0.02$ & $1.40 \pm 0.00$ & 0.76 & 4 & 6 \\
\hline
\end{tabular}


Table 2

(Continued)

\begin{tabular}{|c|c|c|c|c|c|c|c|c|c|}
\hline Name & Packed & $H$ & $G$ & $d(\mathrm{~km})$ & $p_{V}$ & $\eta$ & W2 amp. & $n_{W 1}$ & $n_{W 2}$ \\
\hline 2014 TF64 & K14T64F & 20.10 & 0.15 & $0.70 \pm 0.20$ & $0.03 \pm 0.03$ & $1.40 \pm 0.35$ & 0.33 & 0 & 5 \\
\hline 2014 TJ64 & K14T64J & 21.30 & 0.15 & $0.52 \pm 0.20$ & $0.02 \pm 0.02$ & $1.40 \pm 0.47$ & 0.46 & 0 & 31 \\
\hline 2014 TJ64 & K14T64J & 21.30 & 0.15 & $0.52 \pm 0.23$ & $0.02 \pm 0.03$ & $1.40 \pm 0.54$ & 0.55 & 0 & 14 \\
\hline 2014 UG176 & K14UH6G & 21.50 & 0.15 & $0.42 \pm 0.12$ & $0.03 \pm 0.03$ & $1.40 \pm 0.39$ & 0.17 & 0 & 8 \\
\hline 2014 US192 & K14UJ2S & 18.70 & 0.15 & $0.87 \pm 0.01$ & $0.08 \pm 0.01$ & $1.40 \pm 0.00$ & 0.25 & 5 & 5 \\
\hline 2014 UF206 & K14UK6F & 18.80 & 0.15 & $1.63 \pm 0.79$ & $0.02 \pm 0.04$ & $1.40 \pm 0.49$ & 0.62 & 0 & 17 \\
\hline 2014 UH210 & K14UL0H & 21.10 & 0.15 & $0.40 \pm 0.16$ & $0.04 \pm 0.06$ & $1.40 \pm 0.47$ & 0.76 & 0 & 5 \\
\hline 2014 VP35 & K14V35P & 22.70 & 0.15 & $0.12 \pm 0.05$ & $0.10 \pm 0.10$ & $1.40 \pm 0.53$ & 0.36 & 0 & 6 \\
\hline 2014 WJ70 & K14W70J & 17.60 & 0.15 & $2.92 \pm 1.21$ & $0.02 \pm 0.04$ & $1.40 \pm 0.44$ & 0.62 & 0 & 27 \\
\hline 2014 XQ7 & K14X07Q & 20.60 & 0.15 & $0.65 \pm 0.29$ & $0.02 \pm 0.05$ & $1.40 \pm 0.55$ & 0.83 & 0 & 8 \\
\hline 2014 XX7 & K14X07X & 19.80 & 0.15 & $1.20 \pm 0.38$ & $0.01 \pm 0.02$ & $1.40 \pm 0.36$ & 0.43 & 0 & 6 \\
\hline 2014 XX31 & K14X31X & 17.60 & 0.15 & $1.35 \pm 0.49$ & $0.09 \pm 0.15$ & $1.40 \pm 0.43$ & 0.42 & 0 & 8 \\
\hline
\end{tabular}

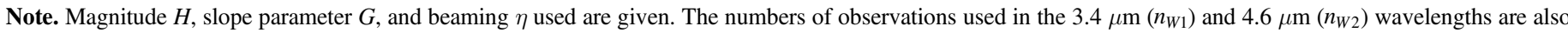
reported, along with the amplitude of the $4.6 \mu \mathrm{m}$ light curve (W2 amp.).

(This table is available in machine-readable form.)

Table 3

Measured Diameters $(d)$ and Albedos $\left(p_{V}\right)$ of Near-Earth Objects Previously Characterized Using NEOWISE Data

\begin{tabular}{|c|c|c|c|c|c|c|c|c|c|}
\hline Name & Packed & $H$ & $G$ & $d(\mathrm{~km})$ & $p_{V}$ & $\eta$ & W2 Amp. & $n_{W 1}$ & $n_{W 2}$ \\
\hline 2102 & 02102 & 16.00 & 0.15 & $1.68 \pm 0.05$ & $0.25 \pm 0.04$ & $1.40 \pm 0.00$ & 0.23 & 13 & 13 \\
\hline 2102 & 02102 & 16.00 & 0.15 & $1.65 \pm 0.05$ & $0.26 \pm 0.04$ & $1.40 \pm 0.00$ & 0.18 & 5 & 5 \\
\hline 2102 & 02102 & 16.00 & 0.15 & $1.69 \pm 0.06$ & $0.25 \pm 0.03$ & $1.40 \pm 0.00$ & 0.67 & 8 & 9 \\
\hline 3554 & 03554 & 15.82 & 0.15 & $1.56 \pm 0.07$ & $0.34 \pm 0.06$ & $1.40 \pm 0.00$ & 0.49 & 19 & 20 \\
\hline 4183 & 04183 & 14.40 & 0.15 & $2.94 \pm 0.12$ & $0.36 \pm 0.06$ & $1.40 \pm 0.00$ & 1.04 & 12 & 12 \\
\hline 4183 & 04183 & 14.40 & 0.15 & $3.54 \pm 0.12$ & $0.24 \pm 0.04$ & $1.40 \pm 0.00$ & 0.62 & 17 & 18 \\
\hline 6050 & 06050 & 14.80 & 0.15 & $2.88 \pm 0.07$ & $0.26 \pm 0.04$ & $1.40 \pm 0.00$ & 1.51 & 57 & 57 \\
\hline 25916 & 25916 & 13.60 & 0.15 & $5.96 \pm 0.13$ & $0.18 \pm 0.03$ & $1.40 \pm 0.00$ & 0.63 & 24 & 29 \\
\hline 27346 & 27346 & 15.90 & 0.15 & $1.80 \pm 0.07$ & $0.24 \pm 0.04$ & $1.40 \pm 0.00$ & 0.43 & 9 & 9 \\
\hline 40263 & 40263 & 17.70 & 0.15 & $0.92 \pm 0.35$ & $0.17 \pm 0.18$ & $1.40 \pm 0.48$ & 0.71 & 0 & 14 \\
\hline 40267 & 40267 & 15.40 & 0.15 & $2.39 \pm 0.09$ & $0.21 \pm 0.04$ & $1.40 \pm 0.00$ & 1.06 & 4 & 4 \\
\hline 85628 & 85628 & 17.00 & 0.15 & $0.78 \pm 0.03$ & $0.46 \pm 0.08$ & $1.40 \pm 0.00$ & 0.64 & 7 & 10 \\
\hline 90367 & 90367 & 17.70 & 0.15 & $1.76 \pm 0.79$ & $0.05 \pm 0.13$ & $1.40 \pm 0.51$ & 0.54 & 0 & 12 \\
\hline 90367 & 90367 & 17.70 & 0.15 & $2.00 \pm 0.89$ & $0.04 \pm 0.03$ & $1.40 \pm 0.46$ & 0.49 & 0 & 13 \\
\hline 137062 & D7062 & 16.60 & 0.15 & $0.99 \pm 0.06$ & $0.41 \pm 0.05$ & $1.40 \pm 0.00$ & 0.89 & 6 & 6 \\
\hline 138847 & D8847 & 16.90 & 0.15 & $0.94 \pm 0.28$ & $0.35 \pm 0.19$ & $1.40 \pm 0.44$ & 1.01 & 0 & 26 \\
\hline 162181 & G2181 & 18.20 & 0.15 & $0.73 \pm 0.02$ & $0.17 \pm 0.03$ & $1.40 \pm 0.00$ & 0.32 & 25 & 25 \\
\hline 162483 & G2483 & 17.50 & 0.15 & $0.69 \pm 0.20$ & $0.37 \pm 0.21$ & $1.40 \pm 0.44$ & 0.62 & 0 & 9 \\
\hline 162566 & G2566 & 15.70 & 0.15 & $6.00 \pm 2.42$ & $0.03 \pm 0.04$ & $1.40 \pm 0.40$ & 1.02 & 0 & 24 \\
\hline 163691 & G3691 & 17.00 & 0.15 & $3.06 \pm 1.55$ & $0.03 \pm 0.06$ & $1.40 \pm 0.54$ & 0.30 & 0 & 5 \\
\hline 243566 & O3566 & 17.40 & 0.15 & $0.88 \pm 0.02$ & $0.25 \pm 0.04$ & $1.40 \pm 0.00$ & 0.29 & 11 & 11 \\
\hline 262623 & Q2623 & 18.50 & 0.15 & $0.49 \pm 0.15$ & $0.29 \pm 0.18$ & $1.40 \pm 0.44$ & 0.48 & 0 & 4 \\
\hline 276049 & R6049 & 16.80 & 0.15 & $4.03 \pm 1.85$ & $0.02 \pm 0.04$ & $1.40 \pm 0.44$ & 0.54 & 0 & 6 \\
\hline 277616 & R7616 & 17.40 & 0.15 & $1.28 \pm 0.01$ & $0.12 \pm 0.02$ & $1.40 \pm 0.00$ & 0.28 & 4 & 4 \\
\hline 395207 & d5207 & 19.60 & 0.15 & $0.60 \pm 0.20$ & $0.07 \pm 0.03$ & $1.40 \pm 0.40$ & 0.32 & 0 & 8 \\
\hline 395207 & d5207 & 19.60 & 0.15 & $0.73 \pm 0.30$ & $0.05 \pm 0.10$ & $1.40 \pm 0.49$ & 0.50 & 0 & 19 \\
\hline 397237 & d7237 & 16.70 & 0.15 & $1.73 \pm 0.66$ & $0.12 \pm 0.16$ & $1.40 \pm 0.46$ & 0.40 & 0 & 4 \\
\hline 1998 SB15 & J98S15B & 20.90 & 0.15 & $0.36 \pm 0.12$ & $0.06 \pm 0.09$ & $1.40 \pm 0.44$ & 0.66 & 0 & 11 \\
\hline 2009 UX17 & K09U17X & 21.50 & 0.15 & $0.39 \pm 0.13$ & $0.03 \pm 0.03$ & $1.40 \pm 0.40$ & 0.86 & 0 & 15 \\
\hline 2010 LF86 & K10L86F & 17.30 & 0.15 & $2.30 \pm 0.89$ & $0.04 \pm 0.04$ & $1.40 \pm 0.41$ & 0.21 & 0 & 7 \\
\hline 2010 LO97 & K10L97O & 18.70 & 0.15 & $1.40 \pm 0.57$ & $0.03 \pm 0.06$ & $1.40 \pm 0.47$ & 0.57 & 0 & 15 \\
\hline 2010 NG3 & K10N03G & 17.20 & 0.15 & $1.45 \pm 0.02$ & $0.11 \pm 0.02$ & $1.40 \pm 0.00$ & 0.64 & 17 & 17 \\
\hline 2010 NG3 & K10N03G & 17.20 & 0.15 & $1.74 \pm 0.94$ & $0.08 \pm 0.18$ & $1.40 \pm 0.59$ & 0.80 & 0 & 17 \\
\hline 2014 HJ129 & K14HC9J & 21.10 & 0.15 & $0.59 \pm 0.21$ & $0.02 \pm 0.02$ & $1.40 \pm 0.42$ & 0.50 & 0 & 9 \\
\hline
\end{tabular}

Note. Objects in this table have previously reported measurements by the NEOWISE team (Mainzer et al. 2011b, 2012). Previous measurements use detections in the 12 and $22 \mu \mathrm{m}$ bands, and therefore are better constrained. Magnitude $H$, slope parameter $G$, and beaming $\eta$ used are given. The numbers of observations used in the $3.4 \mu \mathrm{m}\left(n_{W 1}\right)$ and $4.6 \mu \mathrm{m}\left(n_{W 2}\right)$ wavelengths are also reported, along with the amplitude of the $4.6 \mu \mathrm{m}$ light curve (W2 amp.).

(This table is available in machine-readable form.) 
Table 4

Measured Diameters $(d)$ and Albedos $\left(p_{V}\right)$ of MBAs and Mars Crossers Not Previously Characterized Using NEOWISE Data

\begin{tabular}{|c|c|c|c|c|c|c|c|c|c|}
\hline Name & Packed & $H$ & $G$ & $d(\mathrm{~km})$ & $p_{V}$ & $\eta$ & W2 Amp. & $n_{W 1}$ & $n_{W 2}$ \\
\hline 21 & 00021 & 7.35 & 0.11 & $99.47 \pm 27.12$ & $0.16 \pm 0.12$ & $0.95 \pm 0.19$ & 0.27 & 10 & 10 \\
\hline 65 & 00065 & 6.62 & 0.01 & $276.58 \pm 74.49$ & $0.06 \pm 0.04$ & $0.95 \pm 0.17$ & 0.09 & 8 & 10 \\
\hline 69 & 00069 & 7.05 & 0.19 & $131.07 \pm 32.19$ & $0.19 \pm 0.07$ & $0.95 \pm 0.18$ & 0.09 & 14 & 14 \\
\hline 74 & 00074 & 8.66 & 0.15 & $105.13 \pm 29.95$ & $0.05 \pm 0.02$ & $0.95 \pm 0.16$ & 0.24 & 9 & 9 \\
\hline 140 & 00140 & 8.34 & 0.15 & $82.63 \pm 20.19$ & $0.09 \pm 0.07$ & $0.95 \pm 0.18$ & 0.37 & 7 & 7 \\
\hline 144 & 00144 & 7.91 & 0.17 & $131.36 \pm 33.30$ & $0.05 \pm 0.01$ & $0.95 \pm 0.17$ & 0.31 & 10 & 10 \\
\hline 160 & 00160 & 9.08 & 0.15 & $69.62 \pm 13.23$ & $0.07 \pm 0.04$ & $0.95 \pm 0.14$ & 0.58 & 20 & 21 \\
\hline 212 & 00212 & 8.28 & 0.15 & $132.58 \pm 48.48$ & $0.05 \pm 0.03$ & $0.95 \pm 0.20$ & 0.16 & 5 & 5 \\
\hline 212 & 00212 & 8.28 & 0.15 & $129.09 \pm 40.48$ & $0.05 \pm 0.04$ & $0.95 \pm 0.19$ & 0.17 & 7 & 7 \\
\hline 253 & 00253 & 10.30 & 0.15 & $50.35 \pm 17.16$ & $0.04 \pm 0.02$ & $0.95 \pm 0.24$ & 0.43 & 16 & 16 \\
\hline 284 & 00284 & 10.05 & 0.11 & $54.47 \pm 20.59$ & $0.04 \pm 0.03$ & $0.95 \pm 0.23$ & 0.21 & 11 & 11 \\
\hline 284 & 00284 & 10.05 & 0.11 & $56.81 \pm 15.15$ & $0.04 \pm 0.01$ & $0.95 \pm 0.16$ & 0.25 & 23 & 23 \\
\hline
\end{tabular}

Note. Objects in this table do not have previously published diameters and albedos by the NEOWISE team. Beaming $\eta, H, G$, the amplitude of the $4.6 \mu \mathrm{m}$ light curve (W2 amp.), and the numbers of observations used in the $3.4 \mu \mathrm{m}\left(n_{W 1}\right)$ and $4.6 \mu \mathrm{m}\left(n_{W 2}\right)$ wavelengths are also reported. For a small $(<1 \%)$ fraction of objects, diameter fits could not reproduce optical magnitudes for a realistic range of albedos. This may be due to a large light curve amplitude, uncertainty in $G$ slope values used to derive $H$ magnitudes, or other reasons noted in Mainzer et al. $(2011 \mathrm{~b}, 2012)$, Masiero et al. $(2011,2012)$. These objects are marked with a $\dagger$ in the name column. Objects without reported albedos did not have measured $H$ values, see text for details. Only the first 15 lines are shown.

(This table is available in its entirety in machine-readable form.)

Table 5

Measured Diameters $(d)$ and Albedos $\left(p_{V}\right)$ of MBA and Mars Crossers Previously Characterized Using NEOWISE Data

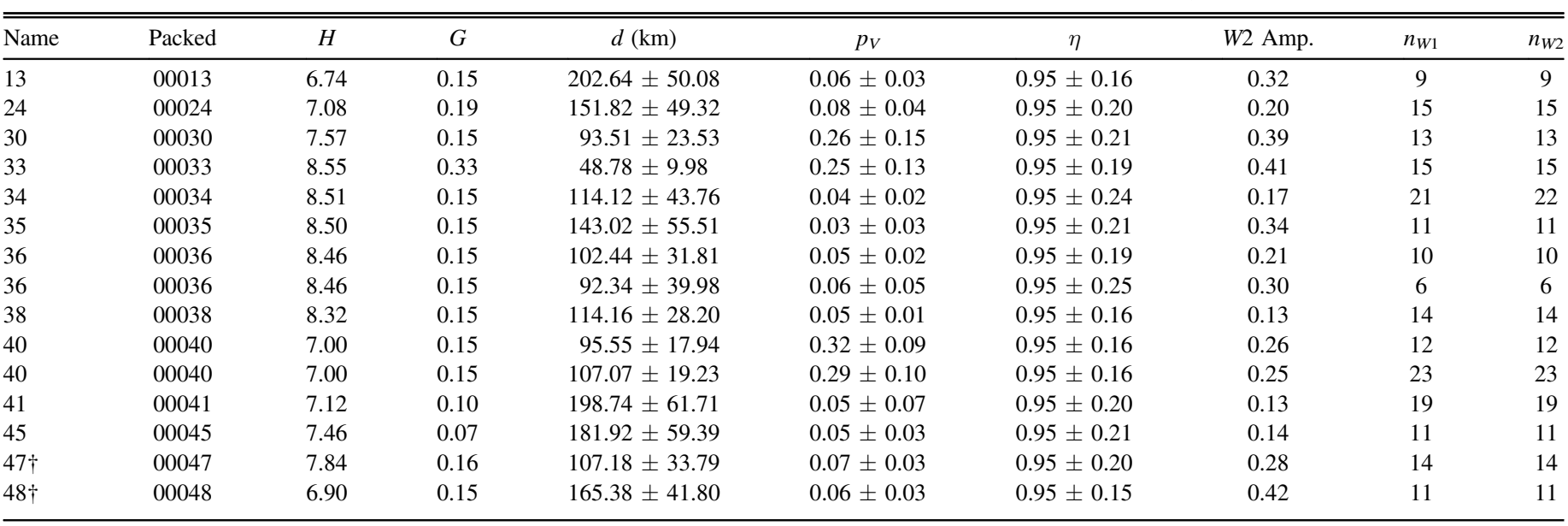

Note. Objects in this table have previously reported measurements by the NEOWISE team (Masiero et al. 2011, 2012). Previous measurements use detections in 12 and $22 \mu \mathrm{m}$ bands, and therefore are better constrained. $H, G$, beaming $\eta$, the amplitude of the $4.6 \mu \mathrm{m}$ light curve (W2 amp.), and the numbers of observations used in the $3.4 \mu \mathrm{m}\left(n_{W 1}\right)$ and $4.6 \mu \mathrm{m}\left(n_{W 2}\right)$ wavelengths are also reported. For a small $(<1 \%)$ fraction of objects, diameter fits could not reproduce optical magnitudes for a realistic range of albedos. This may be due to a large light curve amplitude, uncertainty in $G$ slope values used to derive $H$ magnitudes, or other reasons noted in Mainzer et al. (2011b, 2012), Masiero et al. (2011, 2012). These objects are marked with a $\dagger$ in the name column. Only the first 15 lines are shown.

(This table is available in its entirety in machine-readable form.)

dominated until after the fit was performed. Comparison of those results to NEOWISE fits using $12 \mu \mathrm{m}$ images and radar data confirmed that the thermal fits were poor, so we did not include results that had more than $25 \%$ reflected light in the $4.6 \mu \mathrm{m}$ band.

We assumed that $\eta$ was equal to the average value for the object's population, as determined by Mainzer et al. (2011b) or Masiero et al. (2011), respectively. For NEAs, this meant $\eta=1.4 \pm 0.5$; for all other asteroids in this paper, $\eta=0.95 \pm$ 0.25 . As shown in Masiero et al. (2011), although the average $\eta$ for the main belt is 1.0 , the peak of the histogram is located closer to 0.95 , so this value was adopted in this work.

Following the average values determined by Mainzer et al. (2011b) and Masiero et al. (2011), the ratio of infrared to visible albedo $p_{\mathrm{IR}} / p_{V}$ was initially set to $1.6 \pm 1.0$ for NEAs and $1.5 \pm 0.5$ for Mars-crossers and MBAs. Additionally, it was assumed that the albedos of each band were equal, or $p_{3.4 \mu \mathrm{m}}=p_{4.6 \mu \mathrm{m}}$. Although this may be a poor assumption for objects with red slopes (Grav et al. 2012b), it is necessary to prevent over fitting of the data. 

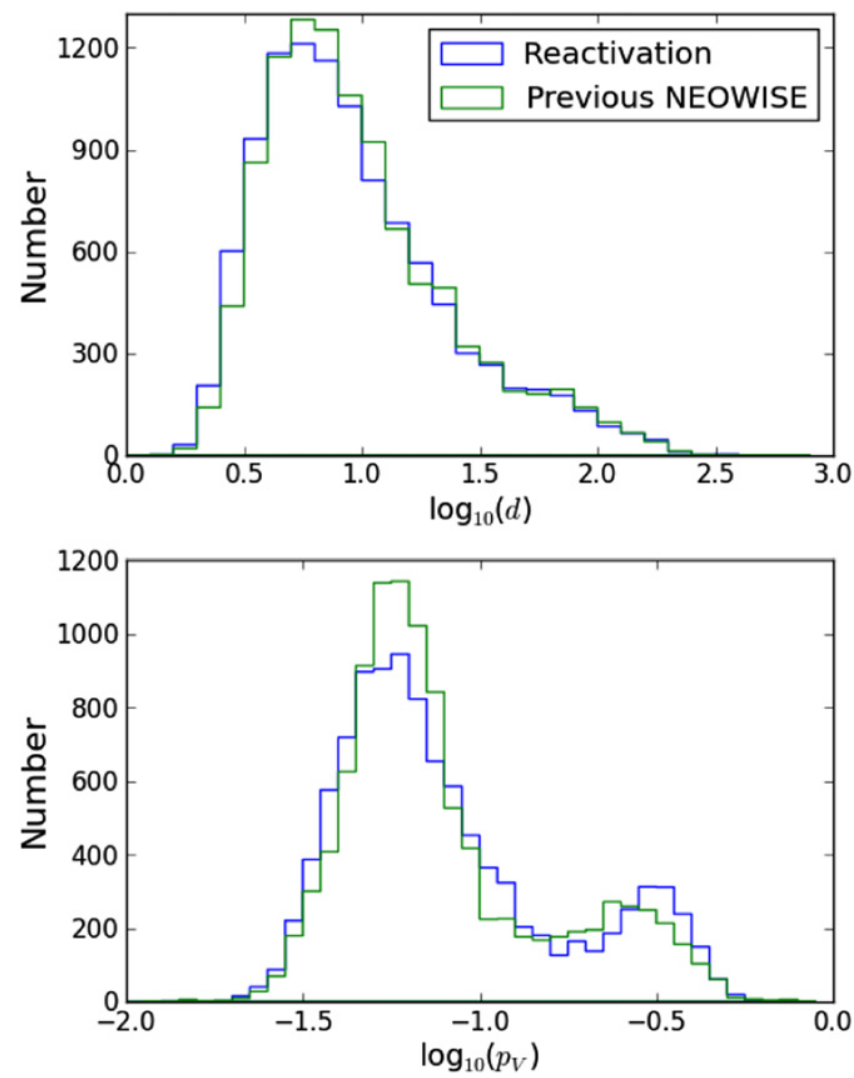

Figure 3. Histogram of MBA diameters (top) and albedos (bottom) measured in this work (blue), and values for the same objects measured by the NEOWISE team previously (green). The two albedo peaks are due to the predominance of bright S-type $\left(p_{V}=0.25\right)$ and dark C-type $\left(p_{V}=0.06\right)$ objects in the Main Belt.

Table 6

Measured Diameters and Albedos for Three Objects Using the Model of Wright (2007)

\begin{tabular}{lcc}
\hline \hline Name & $D(\mathrm{~km})$ & $p_{V}$ \\
\hline 68267 & $0.89 \pm 0.27$ & $0.38 \pm 0.32$ \\
138127 & $0.94 \pm 0.15$ & $0.35 \pm 0.08$ \\
285944 & $1.37 \pm 0.23$ & $0.34 \pm 0.08$ \\
\hline
\end{tabular}

\subsection{Uncertainties}

Uncertainties on $d, p_{V}$, and $\eta$ (when $\eta$ was a free parameter) were determined using a Monte Carlo method. Measured NEOWISE magnitudes, $H$, and $G$ were randomly adjusted within their errors, and the resultant model values of $d, p_{V}$, and (in appropriate cases) $\eta$, were compared to the best-fit values. This process was repeated 25 times for each object, and the resultant errors are the weighted standard deviation of the Monte Carlo trials. The errors quoted in the tables below only include the random component measured through this MC method, not the systematic offset.

Systematic errors were computed by comparing the match between diameters derived in this work to radar-derived diameters for the same objects. Albedos were derived from the radar diameters using the equation

$$
d=\frac{1329}{\sqrt{p_{V}}} 10^{-H / 5}
$$

where $d$ is the diameter in kilometers (for more information, see Harris \& Lagerros 2002).

\subsection{Objects without Visible-wavelength Detections}

Some MBA and Mars-crossing asteroids had no visiblewavelength measurements available from the MPC. Unlike NEAs, objects determined to have these orbits by the MPC are not added to the MPC's NEO Confirmation Page. Therefore, optical follow-up of these objects is rare, and usually serendipitous. For objects without reported optical detections, the $H$ values in MPCORB.dat represent estimates, not measurements, and $p_{V}$ could not be derived. Since thermally emitted light weakly depends on albedo, $d$ measurements are reported for these objects. However, lacking targeted followup, these objects have short arcs and relatively large position uncertainties, which can add additional systematic errors to the derived diameters.

\subsection{NEAs}

NEAs were examined with particular care. Objects with poor agreement between observed and modeled $H$ values were refitted with a parameter that tightened the constraints between modeled and observed $H$. Finally, in some cases an assumption of fixed $\eta=1.4$ produced a poor result. For NEAs with poor fits, beaming was varied between 1.0 and 2.0 , to see if a statistically significant improvement in fit to the observed NEOWISE magnitudes could be achieved.

\section{RESULTS}

Results are divided into four tables. As diameters were calculated using different parameters for the NEAs vs the MBAs and Mars-crossing asteroids, results for these two groups are presented separately. Results are further subdivided between objects that were characterized previously by the NEOWISE team, and objects that were not. This is because previously published values likely used the fully cryogenic 12 and $22 \mu \mathrm{m}$ wavelengths, and therefore can derive diameters more accurately, to within $10 \%$. Researchers looking for the best-constrained diameter and albedo measurements should consult previously published work (Mainzer et al. 2011b, 2012; Masiero et al. 2011, 2012). However, for those researchers who are interested in diameters and albedos derived from additional epochs of data provided by the Year One Reactivation results, we also include the diameters derived for objects using these new data.

Tables 2 and 3 contain the fit diameters and albedos for 173 new and 28 previously characterized NEAs, respectively. Tables 4 and 5 contain the fit diameters and albedos for 1176 new and 6579 previously characterized MBAs and Mars crossing asteroids, respectively. Several objects were observed at multiple apparitions; in these cases, results are presented for each apparition.

Results were compared to previous work by the NEOWISE team (Mainzer et al. 2011b, 2012; Masiero et al. 2011, 2012). Figure 3 shows the comparison between diameter and albedo measurements of MBAs. As observed in Masiero et al. (2011), asteroids in the Main Belt group into bright and dark types, with a greater fraction of bright objects found in the inner regions of the belt. Objects that were also modeled with the thermophysical model of Wright (2007) are given in Table 6. 

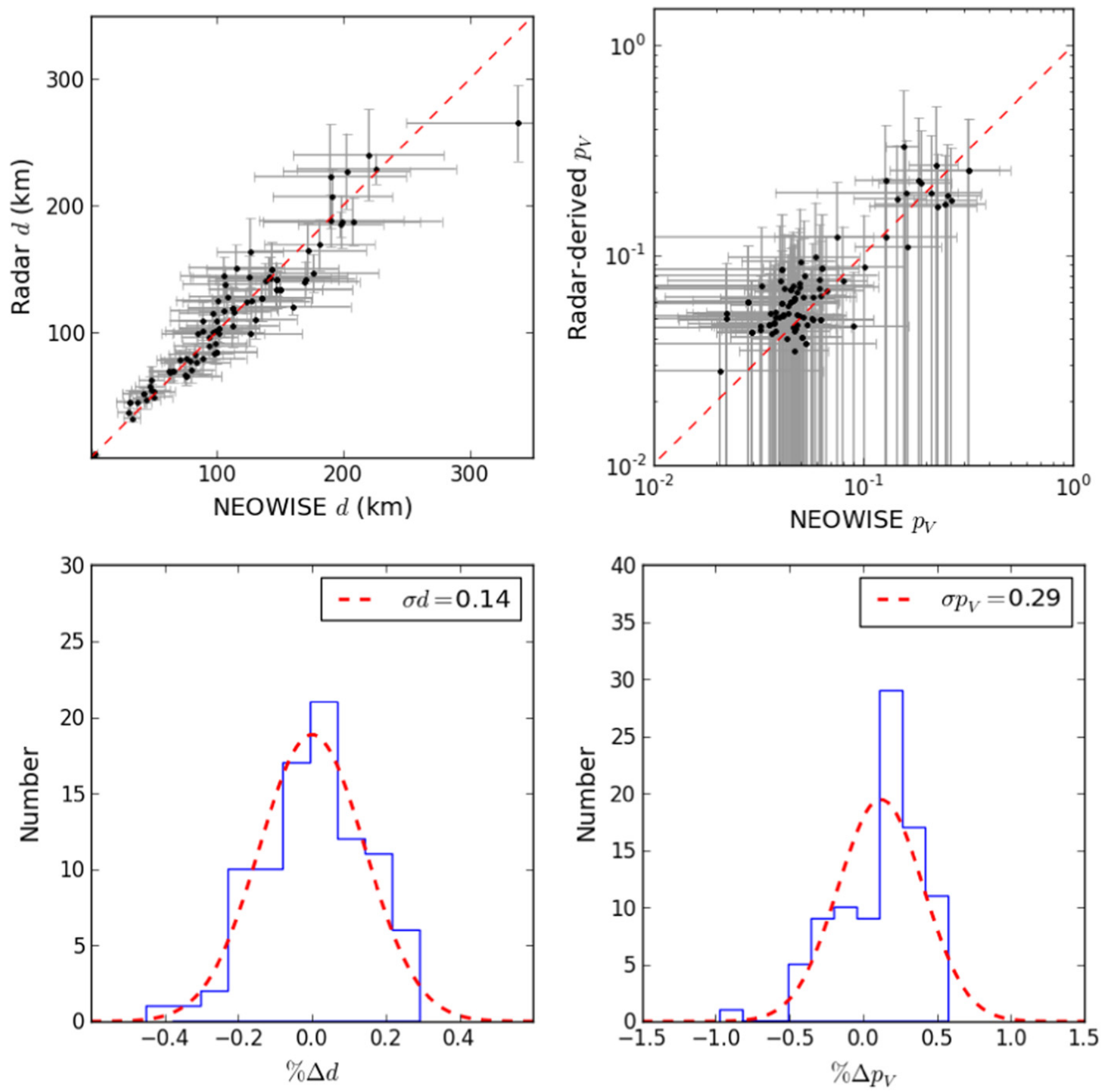

Figure 4. Top: comparison of radar-derived diameters and albedos to the values derived in this paper. The dashed red line shows a 1:1 relation. Bottom: $\% \Delta d$ (left) and $\% \Delta p_{V}$ (right) are the fractional differences between the NEOWISE Reactivation radar-derived diameters and albedos, respectively. The dashed red line is best-fit Gaussian, with the fitted $\sigma$ given in the legends.

When possible, derived diameters were compared to diameter measurements made from radar data. Radar-derived diameters are ideal for this purpose, as they are derived via an independent method (Benner et al. 2015). This comparison is shown in Figure 4. Although the histograms in the figure are not perfectly Gaussian, a best-fit Gaussian to their forms gives fitted $\sigma$ values, which indicates a $14 \%$ relative accuracy in diameter, and a $29 \%$ relative accuracy in albedo. These values are consistent with previous NEOWISE 3-band data results (Mainzer et al. 2012; Masiero et al. 2012). From this comparison to radar-derived diameters and previous work, we conclude that diameters are determined to an accuracy of $\sim 20 \%$ or better. If good-quality $H$ magnitudes are available, albedos can be determined to within $\sim 40 \%$ or better.

Roughly $3 \%$ of objects in this work have significantly different derived diameters than previously published $N E O$ WISE values. It is possible that some of these objects are elongated. NEOWISE collects a sparsely sampled lightcurve for each object, and for example, it is possible that the prime mission happened to observe one of these objects in a more edge-on shape, whereas the reactivation observations tended to observe a wider side of the object. Alternatively, changes in viewing geometry between epochs could result in different diameter measurements; a pole-on viewing geometry could 


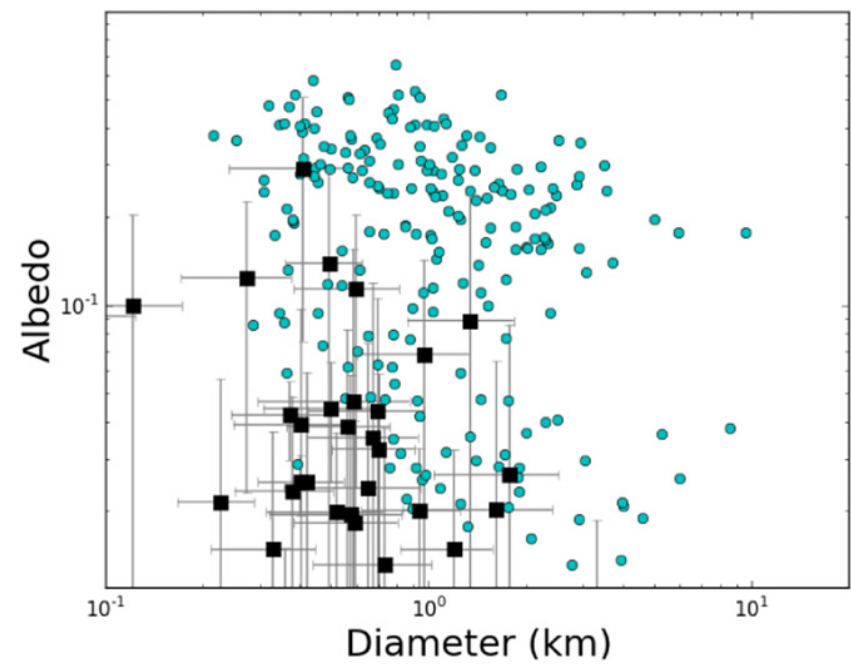

Figure 5. NEOWISE detects large NEAs, and discoveries tend to be dark. Cyan circles are measured diameters and albedos of objects detected in the first year of NEOWISE's Reactivation mission; black squares indicate NEAs discovered by NEOWISE. Error bars on detected objects are omitted for clarity.

Table 7

Measured Diameters and Albedos for Objects that Meet NHATS Criteria. Also Included are the Minimum Round Trip Time in Days, as Determined by the Barbee et al. (2013) Study

\begin{tabular}{lccc}
\hline \hline Name & $D(\mathrm{~km})$ & $p_{V}$ & Minimum Round Trip (days) \\
\hline 1943 Anteros & $2.30 \pm 0.05$ & $0.17 \pm 0.02$ & 354 \\
35107 & $1.00 \pm 0.15$ & $0.34 \pm 0.10$ & 354 \\
363505 & $1.90 \pm 0.05$ & $0.03 \pm 0.01$ & 314 \\
387733 & $0.33 \pm 0.05$ & $0.44 \pm 0.15$ & 354 \\
419624 & $0.35 \pm 0.13$ & $0.09 \pm 0.15$ & 362 \\
\hline
\end{tabular}

have a larger cross section than a geometry aligned with the plane of the equator.

For a small $(<1 \%)$ fraction of objects, diameter fits could not reproduce optical magnitudes for a realistic range of albedos. This may be due to a large light curve amplitude (see column $W 2$ amp. for the amplitude of the $3.4 \mu \mathrm{m}$ band light curve, though note that this is a sparsely sampled light curve), uncertainty in $G$ slope values used to derive $H$ magnitudes, or other reasons noted in Mainzer et al. (2011b, 2012), Masiero et al. (2011, 2012). Poor-quality $H$ values can drive albedo fits to extremes; therefore very low $(\sim 0.01)$ measurements may be signs of this phenomenon.

We have plotted the diameters and albedos of NEOWISE Year One Reactivation discoveries, along with all NEAs detected by NEOWISE (Figure 5). The trend observed in Mainzer et al. (2014) is also present here: NEOWISE tends to discover darker NEAs than optical surveys. This is a direct consequence of the infrared wavelengths that NEOWISE employs.

\subsection{NHATS Targets}

Five objects in this paper meet the NEO Human Space Flight Accessible Targets Study qualifications (Barbee et al. 2013). These objects are listed in Table 7. If an object was observed over multiple epochs, values of $d$ and $p_{V}$ in this table are the averages of the values and associated errors derived at each of those epochs. Asteroid 419624 was discovered in 2010 by NEOWISE.

\section{CONCLUSION}

We present preliminary diameters and albedos for 7956 asteroids observed in the first year of the NEOWISE Reactivation mission. Five of these objects are NHATS targets. Future work by the NEOWISE team includes preliminary characterization results from the continuing mission.

Uncertainties on $d$ and $p_{V}$ are consistent with the errors measured during the initial post-cryo mission. NEOWISE is expected to maintain this pace of detection and NEO discovery for the extent of its mission, currently expected to run through 2017. These results demonstrate the power of infrared survey telescopes to characterize basic physical parameters for large numbers of small bodies.

C.R.N. was partially supported by an appointment to the NASA Postdoctoral Program at the Jet Propulsion Laboratory (JPL), administered by Oak Ridge Associated Universities through a contract with NASA. This publication makes use of data products from the Wide-field Infrared Survey Explorer, which is a joint project of the University of California, Los Angeles, and JPL/California Institute of Technology, funded by NASA. This publication also makes use of data products from NEOWISE, which is a project of the JPL/California Institute of Technology, funded by the Planetary Science Division of NASA. This research has made use of the NASA/ IPAC Infrared Science Archive. The JPL High-Performance Computing Facility used for our simulations is supported by the JPL Office of the CIO.

This project used data obtained with the Dark Energy Camera (DECam), which was constructed by the Dark Energy Survey (DES) collaboration. Funding for the DES Projects has been provided by the U.S. Department of Energy, the U.S. National Science Foundation, the Ministry of Science and Education of Spain, the Science and Technology Facilities Council of the United Kingdom, the Higher Education Funding Council for England, the National Center for Supercomputing Applications at the University of Illinois at Urbana-Champaign, the Kavli Institute of Cosmological Physics at the University of Chicago, the Center for Cosmology and Astro-Particle Physics at the Ohio State University, the Mitchell Institute for Fundamental Physics and Astronomy at Texas A\&M University, Financiadora de Estudos e Projetos, Fundação Carlos Chagas Filho de Amparo à Pesquisa do Estado do Rio de Janeiro, Conselho Nacional de Desenvolvimento Cientifico e Tecnológico and the Ministério da Ciência, Tecnologia e Inovacão, the Deutsche Forschungsgemeinschaft, and the Collaborating Institutions in the Dark Energy Survey. The Collaborating Institutions are Argonne National Laboratory, the University of California at Santa Cruz, the University of Cambridge, Centro de Investigaciones Enérgeticas, Medioambientales y Tecnológicas-Madrid, the University of Chicago, University College London, the DES-Brazil Consortium, the University of Edinburgh, the Eidgenössische Technische Hochschule (ETH) Zürich, Fermi National Accelerator Laboratory, the University of Illinois at Urbana-Champaign, the Institut de Ciències de l'Espai (IEEC/CSIC), the Institut de Física d'Altes Energies, Lawrence Berkeley National Laboratory, the Ludwig-Maximilians Universität München and the associated Excellence Cluster universe, the University of Michigan, the National Optical Astronomy Observatory, the University of Nottingham, the Ohio State University, the University of Pennsylvania, the University of Portsmouth, 
SLAC National Accelerator Laboratory, Stanford University, the University of Sussex, and Texas A\&M University.

This work makes use of observations from the LCOGT network.

Follow-up included observations obtained at the Gemini Observatory, which is operated by the Association of Universities for Research in Astronomy, Inc., under a cooperative agreement with the NSF on behalf of the Gemini partnership: the National Science Foundation (United States), the National Research Council (Canada), CONICYT (Chile), the Australian Research Council (Australia), Ministrio da Cincia, Tecnologia e Inovao (Brazil) and Ministerio de Ciencia, Tecnologa e Innovacin Productiva (Argentina).

We thank the anonymous referee for their thoughtful and thorough consideration of our manuscript.

\section{REFERENCES}

Barbee, B. W., Abell, P. A., Adamo, D. R., et al. 2013, in IAA Planetary Defense Conf. Proc. (Paris: IAA)

Bauer, J. M., Grav, T., Blauvelt, E., et al. 2013, ApJ, 773, 22

Benner, L., Busch, M. B., Giorgini, J. D., Taylor, P. A., \& Margot, J. L. 2015 , in Asteroids IV, ed. P. Michel et al. (Tucson, AZ: Univ. of Arizona Press)

Brown, T. M., Baliber, N., Bianco, F. B., et al. 2013, PASP, 125, 1031

Bus, S. J., \& Binzel, R. P. 2002, Icar, 158, 146

Carruba, V., Domingos, R. C., Nesvorný, D., et al. 2013, MNRAS, 433, 2075

Cruikshank, D. P. 1977, Icar, 30, 224

Cutri, R. M., Mainzer, A., Conrow, T., et al. 2015, Explanatory Supplement to the NEOWISE Data Release Products, 1, http://wise2.ipac.caltech.edu/ docs/release/neowise/expsup

Cutri, R. M., Wright, E. L., Conrow, T., et al. 2012, 1, http://wise2. ipac.caltech.edu/docs/release/allsky/expsup/sec8 1.html

Delbó, M., Harris, A. W., Binzel, R. P., Pravec, P., \& Davies, J. K. 2003, Icar, 166,116

Delbó, M., Walsh, K., Mueller, M., Harris, A. W., \& Howell, E. S. 2011, Icar, 212, 138

Gradie, J., \& Tedesco, E. 1982, Sci, 216, 1405

Grav, T., Mainzer, A. K., Bauer, J. M., Masiero, J. R., \& Nugent, C. R. 2012a, ApJ, 759,49

Grav, T., Mainzer, A. K., Bauer, J. M., Masiero, J. R., \& Nugent, C. R. 2012b, ApJ, 759, 49

Grav, T., Mainzer, A. K., Bauer, J., et al. 2011, ApJ, 742, 40
Grav, T., Mainzer, A. K., Bauer, J., et al. 2012c, ApJ, 744, 197

Hansen, O. L. 1976, AJ, 81, 74

Harris, A. W. 1998, Icar, 131, 291

Harris, A. W., \& Lagerros, J. S. V. 2002, in Asteroids III, Botthe, Jr.., et al. (Tucson, AZ: Univ of Arizona Press), 205

Lebofsky, L. A., Veeder, G. J., Lebofsky, M. J., \& Matson, D. L. 1978, Icar, 35,336

Mainzer, A., Bauer, J., Cutri, R. M., et al. 2014, ApJ, 792, 30

Mainzer, A., Bauer, J., Grav, T., et al. 2011a, ApJ, 731, 53

Mainzer, A., Grav, T., Bauer, J., et al. 2011b, ApJ, 743, 156

Mainzer, A., Grav, T., Masiero, J., et al. 2011c, AJ, 736, 100

Mainzer, A., Grav, T., Masiero, J., et al. 2012, ApJL, 760, L12

Mainzer, A., Usui, F., \& Trilling, D. E. 2015, in Asteroids IV, ed. P. Michel et al. (Tuscon, AZ: Univ. of Arizona Press)

Masiero, J. R., DeMeo, F., Kasuga, T., \& Parker, A. H. 2015, in Asteroids IV, ed. P. Michel et al. (Tuscon, AZ: Univ. of Arizona Press)

Masiero, J. R., Grav, T., Mainzer, A. K., et al. 2014, ApJ, 791, 121

Masiero, J. R., Mainzer, A. K., Bauer, J. M., et al. 2013, ApJ, 770, 7

Masiero, J. R., Mainzer, A. K., Grav, T., et al. 2011, ApJ, 741, 68

Masiero, J. R., Mainzer, A. K., Grav, T., et al. 2012, ApJL, 759, L8

Matter, A., Delbó, M., Ligori, S., Crouzet, N., \& Tanga, P. 2011, Icar, 215, 47

McMillan, R. S. 2007, in IAU Symp. 236, ed. G. B. Valsecchi,

D. Vokrouhlický, \& A. Milani (Cambridge: Cambridge Univ. Press), 329

Milani, A., Cellino, A., Knežević, Z., et al. 2014, Icar, 239, 46

Morrison, D., \& Lebofsky, L. 1979, in Radiometry of Asteroids, ed. T. Gehrels (Tucson, AZ: Univ. of Arizona Press)

Müller, T. G., Miyata, T., Kiss, C., et al. 2013, A\&A, 558, A97

Müller, T. G., O'Rourke, L., Barucci, A. M., et al. 2012, A\&A, 548, A36

Ostro, S. J., Hudson, R. S., Benner, L. A. M., et al. 2002, in Asteroids III, ed. W. F. Botthe Jr. et al. (Tuscon, AZ: Univ. of Arizona Press), 205

Pravec, P., Harris, A. W., Kušnirák, P., Galád, A., \& Hornoch, K. 2012, Icar, 221, 365

Tedesco, E. F., Noah, P. V., Noah, M., \& Price, S. D. 2002, AJ, 123, 1056

Tholen, D. J., Mainzer, A. K., Bauer, J. M., et al. 2014, MPEC, 145

Walsh, K. J., Delbó, M., Bottke, W. F., Vokrouhlický, D., \& Lauretta, D. S. 2013, Icar, 225, 283

Williams, G. V. 2012, PhD thesis, Open Univ. UK

Wolters, S. D., Green, S. F., McBride, N., \& Davies, J. K. 2005, Icar, 175, 92

Wolters, S. D., Green, S. F., McBride, N., \& Davies, J. K. 2008, Icar, 193 535

Wright, E. L. 2007, arXiv:astro-ph/0703085

Wright, E. L., Eisenhardt, P. R. M., Mainzer, A. K., et al. 2010, AJ, 140, 1868

Zellner, B. 1979, in Asteroids, ed. T. Gehrels (Tucson, AZ: Univ. of Arizona Press), 78 